\title{
Upper Bounds on the Degeneracy of the Ground State in Quantum Field Models
}

\author{
Asao Arai and Daiju Funakawa \\ Department of Mathematics, Hokkaido University, Sapporo 060-0810, Japan \\ Correspondence should be addressed to Asao Arai; arai@math.sci.hokudai.ac.jp
}

Received 31 August 2015; Accepted 28 October 2015

Academic Editor: Kamil Brádler

Copyright (C) 2016 A. Arai and D. Funakawa. This is an open access article distributed under the Creative Commons Attribution License, which permits unrestricted use, distribution, and reproduction in any medium, provided the original work is properly cited.

Axiomatic abstract formulations are presented to derive upper bounds on the degeneracy of the ground state in quantum field models including massless ones. In particular, given is a sufficient condition under which the degeneracy of the ground state of the perturbed Hamiltonian is less than or equal to the degeneracy of the ground state of the unperturbed one. Applications of the abstract theory to models in quantum field theory are outlined.

\section{Introduction}

Let $\mathscr{H}$ be a complex Hilbert space with inner product $\langle\cdot, \cdot\rangle$ (complex linear in the right variable) and norm $\|\cdot\|$. For a linear operator $A$ on $\mathscr{H}$, we denote its domain by $D(A)$ and its spectrum by $\sigma(A)$.

Let $H$ be a self-adjoint operator on $\mathscr{H}$ and bounded below. Then, by abstract use of word, we call the infimum of $\sigma(H)$

$$
E_{0}(H):=\inf \sigma(H)
$$

the lowest or minimal energy of $H$ (this name originally comes from the context in quantum physics where $H$ denotes the Hamiltonian of a quantum system). If $E_{0}(H)$ is an eigenvalue of $H$, then $H$ is said to have ground state and a nonzero vector in the eigenspace $\operatorname{ker}\left(H-E_{0}(H)\right)$ is called a ground state of $H$. In that case, the dimension

$$
m(H):=\operatorname{dim} \operatorname{ker}\left(H-E_{0}(H)\right)
$$

of the eigenspace $\operatorname{ker}\left(H-E_{0}(H)\right)$ is called the degeneracy or the multiplicity of the ground state of $H$. If $m(H)=1$, then the ground state is said to be unique. If $m(H) \geq 2$, then the ground state is said to be degenerate.

As is well known, it has been an important issue to determine the degeneracy of the ground state of a given Hamiltonian in quantum physics. For Schrödinger type Hamiltonians in quantum mechanics with finite degrees of freedom and massive Bose field Hamiltonians in quantum field theory, general theorems on the uniqueness of the ground state and upper bounds on the degeneracy of the ground state have been established (see, e.g., [1, §XIII.12] and references in Notes for $\$$ XIII.12). For fermion systems, Faris [2] discussed conditions which ensure the uniqueness of the ground state. Faris's ideas and methods have been extended by Miyao $[3,4]$ to obtain general criteria on the uniqueness of the ground state in bosonic quantum field models as well as fermionic ones.

As for models in which a massless quantum field appears, estimation of the degeneracy of the ground state is highly nontrivial, because, in that case, one has to treat an embedded eigenvalue problem so that the regular perturbation theory or the min-max principle cannot be used (for a review of this aspect, see, e.g., [5]). A first breakthrough result on this problem was given by Bach et al. [6]. They considered a model in nonrelativistic quantum electrodynamics and proved that, under suitable hypotheses, the degeneracy of the ground state of the total Hamiltonian of the model is less than or equal to the degeneracy of the ground state of the unperturbed Hamiltonian [6, Theorem I.1(f)]. The methods used in [6] to estimate the degeneracy of the ground state have been generalized by Hiroshima [7] to be applied to a class of quantum field models whose Hamiltonian is of the following form:

$$
H(g)=A \otimes I+I \otimes d \Gamma(S)+g H_{\mathrm{int}}
$$


acting in the tensor product Hilbert space

$$
\mathscr{F}:=\mathfrak{h} \otimes \mathscr{F}_{\mathrm{b}}(W)
$$

of a complex Hilbert space $\mathfrak{h}$ and the boson Fock space $\mathscr{F}_{\mathrm{b}}(W)$ over a complex Hilbert space $W$ (see (80) for the definition). Here $A$ is a self-adjoint operator on $\mathfrak{h}$ which is bounded below, $I$ denotes identity, $S$ is a nonnegative selfadjoint operator on $W, d \Gamma(S)$ is the second quantization operator of $S$ on $\mathscr{F}_{\mathrm{b}}(W)$ (see, e.g., [8, p.302] and [9, $₫$ X.7]), $g \in \mathbb{R}$ is a coupling constant, and $H_{\text {int }}$ is a symmetric operator on $\mathscr{F}$ which describes an interaction between a Bose field and a quantum system whose Hilbert space of state vectors is $\mathfrak{h}$. In [7], it is assumed that $H_{\mathrm{int}}$ is relatively bounded with respect to the unperturbed operator $H(0):=A \otimes I+I \otimes$ $d \Gamma(S)$. It is proved in [7] that, under a suitable condition, $m(H(g)) \leq c(g) m(H(0))$ with $c(g)>0$ being a constant depending on $g$ and, in particular, $m(H(g)) \leq m(H(0))$ for all sufficiently small $|g|$ in an abstract framework and in the case where $W=\bigoplus^{D} L^{2}\left(\mathbb{R}^{d}\right)$, the $D$-direct sum of $L^{2}\left(\mathbb{R}^{d}\right)$ $(D, d \in \mathbb{N})$. Moreover, these results were applied to the generalized spin-boson model [10], the Pauli-Fierz model, and a model in relativistic quantum electrodynamics with cutoffs [7].

One of the motivations of the present work comes from extending results in [7] to the case where $H_{\text {int }}$ is not necessarily relatively bounded with respect to $H(0)$. But we find that, before going on analyzing such models, it is better to construct an abstract theory on the degeneracy of ground state with the requirement that it formulates general aspects independent of concrete models. From this point of view, we construct in this paper such an abstract theory in axiomatic manners. Careful investigations and structural analyses on results on the existence and the degeneracy of ground state which have been established so far (e.g., $[6,7,10-$ 12]) make it possible. Applications of the abstract theory to concrete models will be discussed in a separate paper. We hope that the abstract theory given in the present paper not only clarifies general structures behind the theory on the degeneracy of ground state in [7] but also makes the range of applications wider, because the abstract results established in the present paper show what are general independently of models and what should be proved in each concrete model.

The present paper is organized as follows. In Section 2, we consider a bounded below and self-adjoint operator $H_{0}$ and a symmetric operator $H_{1}$ on a Hilbert space $\mathscr{H}$. The sum $H:=$ $H_{0}+H_{1}$ is supposed to be self-adjoint and bounded below. We formulate a sufficient condition which yields inequality $m(H) \leq c m\left(H_{0}\right)$ with a constant $c>0$ being computed from the given data. In particular, an additional condition for $c=1$ is given so that $m(H) \leq m\left(H_{0}\right)$. In Section 3, we derive an integral equation for any ground state of $H$ in terms of a linear operator $A$ on $\mathscr{H}$ which has some characteristic properties (Theorem 11). In Section 4 we state and prove the main theorem in the present paper (Theorem 12). In the last section, we give remarks for applications of the main theorem to concrete models in quantum field theory.

\section{Comparison Theorem on Degeneracy of Ground States}

For nonnegative self-adjoint operators $A_{0}, A_{1}, \ldots, A_{n}$ on $\mathscr{H}$ $(n=1,2, \ldots)$, we write “ $A_{0} \preceq \sum_{j=1}^{n} A_{j}$ ” if $\bigcap_{j=1}^{n} D\left(A_{j}^{1 / 2}\right) \subset$ $D\left(A_{0}^{1 / 2}\right)$ and

$$
\begin{aligned}
\left\langle A_{0}^{1 / 2} \psi, A_{0}^{1 / 2} \psi\right\rangle \leq \sum_{j=1}^{n}\left\langle A_{j}^{1 / 2} \psi, A_{j}^{1 / 2} \psi\right\rangle & \\
& \psi \in \bigcap_{j=1}^{n} D\left(A_{j}^{1 / 2}\right) .
\end{aligned}
$$

Let $H_{0}$ be a bounded below and self-adjoint operator on $\mathscr{H}$ and let $H_{1}$ be a symmetric operator on $\mathscr{H}$ such that

$$
H:=H_{0}+H_{1}
$$

is self-adjoint and bounded below $\left(D(H):=D\left(H_{0}\right) \cap D\left(H_{1}\right)\right)$. We assume the following.

Hypothesis 1. (i) The operators $H_{0}$ and $H$ have ground state with $m\left(H_{0}\right)<\infty$.

(ii) There exists a nonnegative self-adjoint operator $L$ on $\mathscr{H}$ such that

$$
I \preceq L+P_{H_{0}},
$$

where, for a bounded below self-adjoint operator $T$ on $\mathscr{H}$, we denote by $P_{T}$ the orthogonal projection onto $\operatorname{ker}\left(T-E_{0}(T)\right)$ : $P_{T} \mathscr{H}=\operatorname{ker}\left(T-E_{0}(T)\right)$.

A vector $\psi \in \mathscr{H}$ is said to overlap with a subset $D \subset \mathscr{H}$ if there exists a vector $\eta \in D$ such that $\langle\psi, \eta\rangle \neq 0$.

The following theorem, which is a comparison theorem on $m(H)$ and $m\left(H_{0}\right)$, and Corollary 2 below are more abstract versions of Lemma 3.3 and Corollaries 3.4-3.5 in [7].

Theorem 1. Assume Hypothesis 1 and suppose that there exists a constant $\delta$ with $0<\delta<1$ such that

$$
\sup _{\phi \in D\left(L^{1 / 2}\right) \cap P_{H} \mathscr{H},\|\phi\|=1}\left\|L^{1 / 2} \phi\right\|^{2} \leq \delta .
$$

Then,

(i) any $\phi \in P_{H} \mathscr{H}$ with $\|\phi\|=1$ overlaps with $P_{H_{0}} \mathscr{H}$;

(ii) let

$$
m_{L}(H):=\operatorname{dim}\left[D\left(L^{1 / 2}\right) \cap P_{H} \mathscr{H}\right]
$$

then

$$
m_{L}(H) \leq \frac{1}{1-\delta} m\left(H_{0}\right)
$$

(iii) if $P_{H} \mathscr{H} \subset D\left(L^{1 / 2}\right)$ in addition, then

$$
m(H) \leq \frac{1}{1-\delta} m\left(H_{0}\right) .
$$


Proof. (i) Let $\phi \in D\left(L^{1 / 2}\right) \cap P_{H} \mathscr{H}$ with $\|\phi\|=1$. Then, by Hypothesis 1(ii), we have

$$
1 \leq\left\|L^{1 / 2} \phi\right\|^{2}+\left\langle\phi, P_{H_{0}} \phi\right\rangle \leq \delta+\left\langle\phi, P_{H_{0}} \phi\right\rangle .
$$

Hence

$$
1 \leq(1-\delta)^{-1}\left\langle\phi, P_{H_{0}} \phi\right\rangle .
$$

In particular, $\left\langle\phi, P_{H_{0}} \phi\right\rangle \neq 0$. Therefore $\phi$ overlaps with $P_{H_{0}} \mathscr{H}$.

(ii) We first show that $D\left(L^{1 / 2}\right) \cap P_{H} \mathscr{H}$ is finitedimensional. So suppose that $D\left(L^{1 / 2}\right) \cap P_{H} \mathscr{H}$ were infinitedimensional. Then there is an orthonormal system $\left\{\phi_{n}\right\}_{n=1}^{\infty}$ with $\phi_{n} \in D\left(L^{1 / 2}\right) \cap P_{H} \mathscr{H}, n \geq 1$. Taking $\phi_{n}$ as $\phi$ in (13) and summing over $n$, we obtain $\sum_{n=1}^{\infty}\left\langle\phi_{n}, P_{H_{0}} \phi_{n}\right\rangle=\infty$. But the left hand side is less than or equal to $\operatorname{Tr} P_{H_{0}}=m\left(H_{0}\right)<\infty$, where $\operatorname{Tr}$ means trace. Hence one is led to a contradiction. Therefore $D\left(L^{1 / 2}\right) \cap P_{H} \mathscr{H}$ is finite-dimensional; that is, $m_{L}(H)<\infty$. Let $\left\{\phi_{n}\right\}_{n=1}^{m_{L}(H)}$ be an orthonormal basis of $D\left(L^{1 / 2}\right) \cap P_{H} \mathscr{H}$. Then, by (13), we have

$$
\begin{aligned}
m_{L}(H) & \leq \frac{1}{1-\delta} \sum_{n=1}^{m_{L}(H)}\left\langle\phi_{n}, P_{H_{0}} \phi_{n}\right\rangle \leq \frac{1}{1-\delta} \operatorname{Tr} P_{H_{0}} \\
& =\frac{1}{1-\delta} m\left(H_{0}\right) .
\end{aligned}
$$

Thus (10) follows.

(iii) This follows from (10).

Corollary 2. Assume Hypothesis 1 and suppose that there exists a constant $\delta$ such that

$$
0<\delta<\frac{1}{m\left(H_{0}\right)+1}
$$

and (8) holds. Then

$$
m_{L}(H) \leq m\left(H_{0}\right) .
$$

In particular, if $P_{H} \mathscr{H} \subset D\left(L^{1 / 2}\right)$ in addition, then

$$
m(H) \leq m\left(H_{0}\right) .
$$

Proof. Condition (15) implies that $0<\delta<1$. Hence the conclusion of Theorem 1 holds. Therefore

$$
m_{L}(H) \leq \frac{1}{1-\delta} m\left(H_{0}\right)<m\left(H_{0}\right)+1,
$$

where, in the second inequality, we have used (15). Since $m_{L}(H)$ and $m\left(H_{0}\right)$ are natural numbers, (16) follows.

Corollary 3 (uniqueness). Assume Hypothesis 1 with $m\left(H_{0}\right)$ $=1$ and suppose that the following ( $i$ ) and (ii) hold:

(i) $P_{H} \mathscr{H} \subset D\left(L^{1 / 2}\right)$.

(ii) There exists a constant $\delta$ with $0<\delta<1 / 2$ such that (8) holds.

Then $m(H)=1$.
Proof. In the present case, (15) holds with $m\left(H_{0}\right)=1$. Hence $m(H) \leq 1$. Since $m(H)$ is a natural number, it follows that $m(H)=1$.

In applications to quantum field models, it may be convenient to consider $H$ in the form

$$
H_{g}:=H_{0}+g H_{1}
$$

with a constant parameter $g>0$, as in the case of $H(g)$ given by (3).

Remark 4. The condition $g>0$ is taken so that $H_{g}$ is bounded below in the case where $H_{1}$ is not $H_{0}$-bounded but bounded below. In the case where $H_{1}$ is $H_{0}$-bounded, $g$ needs not to be positive, being allowed to be a negative number as well, and, by the Kato-Rellich theorem, $H_{g}$ is self-adjoint and bounded below for all sufficiently small $|g|(g \in \mathbb{R})[7]$.

Corollary 5. Assume Hypothesis 1 with $H=H_{g}, 0<g<a$ ( $a>0$ is a constant), and suppose that there exists a continuous function $\delta:=\delta(g)$ on $[0, a)$ such that $\delta(0)=0$ and

$$
\sup _{\phi \in D\left(L^{1 / 2}\right) \cap P_{H_{g}} \mathscr{H},\|\phi\|=1}\left\|L^{1 / 2} \phi\right\|^{2} \leq \delta(g), \quad g \in[0, a) .
$$

Then there exists a constant $g_{0} \in(0, a)$ such that, for all $g \in$ $\left(0, g_{0}\right)$, any $\phi \in P_{H_{g}} \mathscr{H}$ with $\|\phi\|=1$ overlaps with $P_{H_{0}} \mathscr{H}$ and

$$
m_{L}\left(H_{g}\right) \leq m\left(H_{0}\right) .
$$

In particular, if $P_{H_{g}} \mathscr{H} \subset D\left(L^{1 / 2}\right)$ for all $g \in\left(0, g_{0}\right)$ in addition, then

$$
m\left(H_{g}\right) \leq m\left(H_{0}\right)
$$

for all $g \in\left(0, g_{0}\right)$.

Proof. By the continuity of $\delta(g)$ in $g \in[0, a)$ with $\delta(0)=0$, there exists a constant $g_{0} \in(0, a)$ such that, for all $g \in\left[0, g_{0}\right)$,

$$
0 \leq \delta(g)<\frac{1}{m\left(H_{0}\right)+1} .
$$

Hence, for each $g \in\left(0, g_{0}\right)$, one can apply Theorem 1 and Corollary 2 to obtain the desired results.

Remark 6. Condition (20) with $g=0$ implies that $D\left(L^{1 / 2}\right) \cap$ $P_{H_{0}} \mathscr{H} \subset \operatorname{ker} L^{1 / 2}=\operatorname{ker} L$. In some models in quantum field theory, $L$ can be taken in such a way that this property is satisfied (see Section 5).

\section{An Abstract Integral Equation for Ground States}

In applications of Theorem 1, we need to prove (8). In concrete models in quantum field theory, this has been done by using operators which have some characteristic relations to $L$ and $H_{0}$. In this section we introduce an abstract version of such operators. 

and

Let $A$ be a densely defined closed linear operator on $\mathscr{H}$

$$
\begin{aligned}
A_{0}(t) & :=e^{i t H_{0}} A e^{-i t H_{0}}, \\
A(t) & :=e^{i t H} A_{0}(-t) e^{-i t H}, \quad t \in \mathbb{R} .
\end{aligned}
$$

We assume the following:

(A.1)

(i) For some $\alpha \in(0,1], A$ is $H_{0}^{\alpha}$-bounded; that is, $D\left(H_{0}^{\alpha}\right) \subset D(A)$, and there exist constants $a, b \geq$ 0 such that

$$
\|A \psi\| \leq a\left\|H_{0}^{\alpha} \psi\right\|+b\|\psi\|, \quad \psi \in D\left(H_{0}^{\alpha}\right) .
$$

(ii) There exists a core $\mathscr{D}$ of $H_{0}^{\alpha}$ such that

$$
\lim _{t \rightarrow \infty} A e^{i t H_{0}} \psi=0, \quad \psi \in \mathscr{D} .
$$

Remark 7. Under condition (A.1)(i), the functional calculus of the self-adjoint operator $H_{0}$ gives that, for all $\psi \in D\left(H_{0}^{\alpha}\right)$ and $t \in \mathbb{R}, e^{i t H_{0}} \psi \in D\left(H_{0}^{\alpha}\right)$ and $H_{0}^{\alpha} e^{i t H_{0}} \psi=e^{i t H_{0}} H_{0}^{\alpha} \psi$. Hence $D\left(H_{0}^{\alpha}\right) \subset D\left(A_{0}(t)\right)$ for all $t \in \mathbb{R}$. In particular, $D\left(H_{0}\right) \subset$ $D\left(A_{0}(t)\right)$ for all $t \in \mathbb{R}$. Since $D(H) \subset D\left(H_{0}\right)$ and $e^{-i t H} D(H)=$ $D(H)$ for all $t \in \mathbb{R}$, it follows that $D(H) \subset D(A(t))$ for all $t \in \mathbb{R}$. In what follows, we use these facts without mentioning.

Lemma 8. Assume (A.1). Then, for all $\phi \in P_{H} \mathscr{H}$, $\lim _{t \rightarrow \infty} A(t) \phi=0$.

Proof. Since $e^{-i t H} \phi=e^{-i t E_{0}(H)} \phi$, we have $\|A(t) \phi\|=$ $\left\|A e^{i t H_{0}} \phi\right\|$. By (A.1)(ii), for any $\varepsilon>0$, there exists a vector $\psi_{\varepsilon} \in \mathscr{D}$ such that

$$
\begin{array}{r}
\left\|\psi_{\varepsilon}-\phi\right\|<\varepsilon, \\
\left\|H_{0}^{\alpha} \psi_{\varepsilon}-H_{0}^{\alpha} \phi\right\|<\varepsilon .
\end{array}
$$

Hence

$$
\begin{aligned}
\left\|A e^{i t H_{0}} \phi\right\| \leq & \left\|A e^{i t H_{0}}\left(\phi-\psi_{\varepsilon}\right)\right\|+\left\|A e^{i t H_{0}} \psi_{\varepsilon}\right\| \\
\leq & a\left\|H_{0}^{\alpha}\left(\phi-\psi_{\varepsilon}\right)\right\|+b\left\|\phi-\psi_{\varepsilon}\right\| \\
& +\left\|A e^{i t H_{0}} \psi_{\varepsilon}\right\|<(a+b) \varepsilon+\left\|A e^{i t H_{0}} \psi_{\varepsilon}\right\| .
\end{aligned}
$$

Taking the limit $t \rightarrow \infty$, we have

$$
0 \leq \limsup _{t \rightarrow \infty}\left\|A e^{i t H_{0}} \phi\right\| \leq(a+b) \varepsilon .
$$

Then the limit $\varepsilon \rightarrow 0$ yields that $\lim _{t \rightarrow \infty}\left\|A e^{i t H_{0}} \phi\right\|=0$. Thus $\lim _{t \rightarrow \infty}\|A(t) \phi\|=0$.

To state additional assumptions, we recall the concept of weak commutator [13]. Let $T$ and $S$ be densely defined linear operators on $\mathscr{H}$ and let $\mathscr{E}$ be a dense subspace in $\mathscr{H}$ such that $\mathscr{E} \subset D(T) \cap D(S) \cap D\left(T^{*}\right) \cap D\left(S^{*}\right)$, where, for a densely defined linear operator $A$ on $\mathscr{H}, A^{*}$ denotes the adjoint of $A$. Then the pair $(T, S)$ is said to have weak commutator on $\mathscr{E}$ if there exists a linear operator $C$ on $\mathscr{H}$ such that $\mathscr{E} \subset D(C)$ and $\left\langle T^{*} \psi, S \phi\right\rangle-$ $\left\langle S^{*} \psi, T \phi\right\rangle=\langle\psi, C \phi\rangle, \psi, \phi \in \mathscr{E}$. In this case, the operator $C$ restricted to $\mathscr{E}$ is written as $[T, S]_{\mathrm{w}}^{\mathscr{E}}$ so that

$$
\left\langle T^{*} \psi, S \phi\right\rangle-\left\langle S^{*} \psi, T \phi\right\rangle=\left\langle\psi,[T, S]_{\mathrm{w}}^{\mathscr{E}} \phi\right\rangle,
$$

$\psi, \phi \in \mathscr{E}$.

We call $[T, S]_{\mathrm{w}}^{\mathscr{E}}$ the weak commutator of $(T, S)$ on $\mathscr{E}$.

We also need the following assumption:

(i) For each $t \in \mathbb{R}$ and $j=0,1,\left(H_{j}, A_{0}(t)\right)$ has weak commutator $\left[H_{j}, A_{0}(t)\right]_{\mathrm{w}}^{D(H)}$ on $D(H)$. Moreover, for all $\psi \in D(H),\left[H_{1}, A_{0}(t)\right]_{\mathrm{w}}^{D(H)} \psi$ is strongly continuous in $t$.

(ii) For all $\psi \in D(H), A_{0}(t) \psi$ is strongly differentiable in $t$ and its strong derivative $d A_{0}(t) \psi / d t$ is of the form

$$
\frac{d A_{0}(t) \psi}{d t}=i\left[H_{0}, A_{0}(t)\right]_{\mathrm{w}}^{D(H)} \psi, \quad t \in \mathbb{R} .
$$

(iii) There exist a $\sigma$-finite measure space $(M, \Sigma, \mu)$, a nonnegative $\Sigma$-measurable function $u$ on $M$ with $0<u(k)<\infty$ for $\mu$-a.e. $k \in M$, and a linear operator $S(k)$ on $\mathscr{H}(k \in M)$ with $D(S(k))$ ว $D(H)$ such that the following (a)-(c) hold:

(a) for all $\psi, \chi \in D(H), \int_{M}|\langle\chi, S(k) \psi\rangle| d \mu(k)<$ $\infty$ and

$$
\begin{aligned}
\langle\chi & {\left.\left[H_{1}, A_{0}(-t)\right]_{\mathrm{w}}^{D(H)} \psi\right\rangle } \\
& =\int_{M} e^{i t u(k)}\langle\chi, S(k) \psi\rangle d \mu(k) ;
\end{aligned}
$$

(b) for all $\phi \in P_{H} \mathscr{H}, \int_{M}\|S(k) \phi\| d \mu(k)<\infty$ and $\int_{M}\|S(k) \phi\| / u(k) d \mu(k)<\infty$;

(c) for all $\psi \in \mathscr{D}$ and $\phi \in P_{H} \mathscr{H}$,

$$
\int_{0}^{\infty}\left|\int_{M}\left\langle\psi, e^{i s\left(H-E_{0}(H)+u(k)\right)} S(k) \phi\right\rangle d \mu(k)\right| d s<\infty .
$$

Remark 9. Condition (a) in (A.2)(iii) may be regarded as an abstract form of "sum rules" in quantum mechanics [13]. In fact, under stronger additional conditions, one can prove (32) (cf. [13]), where $\mu$ is determined by the spectral measure of $\mathrm{H}_{0}$.

Under condition (A.2), $\left(H-E_{0}(H)+u(k)\right)^{-1}$ is bounded for $\mu$-a.e. $k \in M$ with

$$
\left\|\left(H-E_{0}(H)+u(k)\right)^{-1}\right\| \leq \frac{1}{u(k)}, \quad \mu \text {-a.e. } k \in M .
$$

Hence, for all $\phi \in P_{H} \mathscr{H}$,

$$
\begin{gathered}
\int_{M}\left\|\left(H-E_{0}(H)+u(k)\right)^{-1} S(k) \phi\right\| d \mu(k) \\
\leq \int_{M} \frac{\|S(k) \phi\|}{u(k)} d \mu(k)<\infty .
\end{gathered}
$$


It is easy to see that, for all $\psi \in D(H), e^{i s\left(H-E_{0}(H)\right)}\left[H_{1}\right.$, $\left.A_{0}(-s)\right]_{\mathrm{w}}^{D(H)} \psi$ is strongly continuous in $s$. Hence, for each $t \in \mathbb{R}$, the strong Riemann integral $\int_{0}^{t} e^{i s\left(H-E_{0}(H)\right)} \cdot\left[H_{1}\right.$, $\left.A_{0}(-s)\right]_{\mathrm{w}}^{D(H)} \psi d s$ exists.

Lemma 10. Assume (A.1) and (A.2). Then, for all $\phi \in P_{H} \mathscr{H}$ and $t \in \mathbb{R}$,

$$
\begin{aligned}
A(t) \phi= & A \phi \\
& +i \int_{0}^{t} e^{i s\left(H-E_{0}(H)\right)}\left[H_{1}, A_{0}(-s)\right]_{\mathrm{w}}^{D(H)} \phi d s .
\end{aligned}
$$

Moreover, for all $\psi \in D(H)$,

$$
\begin{aligned}
& \langle\psi, A(t) \phi\rangle \\
& =\langle\psi, A \phi\rangle \\
& \quad+i \int_{0}^{t} d s \int_{M} d \mu(k)\left\langle\psi, e^{i s\left(H-E_{0}(H)+u(k)\right)} S(k) \phi\right\rangle .
\end{aligned}
$$

Proof. Let $\chi \in D(H)$ and

$$
f(t):=\langle\chi, A(t) \phi\rangle=\left\langle e^{-i t\left(H-E_{0}(H)\right)} \chi, A_{0}(-t) \phi\right\rangle .
$$

Then it is easy to see that $f(t)$ is differentiable in $t$ with

$$
\begin{aligned}
\frac{d f(t)}{d t}= & i\left\langle\left(H-E_{0}(H)\right) e^{-i t H} \chi, A_{0}(-t) e^{-i t H} \phi\right\rangle \\
& +\left\langle e^{-i t H} \chi,-i\left[H_{0}, A_{0}(-t)\right]_{\mathrm{w}}^{D(H)} e^{-i t H} \phi\right\rangle .
\end{aligned}
$$

Using the identity $\left\langle A_{0}(-t)^{*} e^{-i t H} \chi,\left(H-E_{0}(H)\right) e^{-i t H} \phi\right\rangle=0$, we obtain

$$
\begin{aligned}
\frac{d f(t)}{d t} & =i\left\langle e^{-i t H} \chi,\left[H_{1}, A_{0}(-t)\right]_{\mathrm{w}}^{D(H)} e^{-i t H} \phi\right\rangle \\
& =i\left\langle\chi, e^{i t\left(H-E_{0}(H)\right)}\left[H_{1}, A_{0}(-t)\right]_{\mathrm{w}}^{D(H)} \phi\right\rangle .
\end{aligned}
$$

Hence

$$
\begin{aligned}
f(t) & \\
= & \langle\chi, A \phi\rangle \\
& +\int_{0}^{t} i\left\langle\chi, e^{i s\left(H-E_{0}(H)\right)}\left[H_{1}, A_{0}(-s)\right]_{\mathrm{w}}^{D(H)} \phi\right\rangle d s .
\end{aligned}
$$

By a property of strong Riemann integral, we have

$$
\begin{aligned}
& \int_{0}^{t} i\left\langle\chi, e^{i s\left(H-E_{0}(H)\right)}\left[H_{1}, A_{0}(-s)\right]_{\mathrm{w}}^{D(H)} \phi\right\rangle d s \\
& \quad=\left\langle\chi, i \int_{0}^{t} e^{i s\left(H-E_{0}(H)\right)}\left[H_{1}, A_{0}(-s)\right]_{\mathrm{w}}^{D(H)} \phi d s\right\rangle .
\end{aligned}
$$

Thus (36) follows. Formula (37) follows from (36) and (A.2)(iii).
For all $\phi \in P_{H} \mathscr{H}$,

$$
\begin{gathered}
\int_{M}\left\|\left(H-E_{0}(H)+u(k)\right)^{-1} S(k) \phi\right\| d \mu(k) \\
\leq \int_{M} \frac{\|S(k) \phi\|}{u(k)} d \mu(k)<\infty .
\end{gathered}
$$

Hence the Bochner integral $\int_{M}\left(H-E_{0}(H)+\right.$ $u(k))^{-1} S(k) \phi d \mu(k)$ exists. The main theorem in this section is as follows.

Theorem 11. Assume (A.1) and (A.2). Then, for all $\phi \in P_{H} \mathscr{H}$,

$$
A \phi=\int_{M}\left(H-E_{0}(H)+u(k)\right)^{-1} S(k) \phi d \mu(k) .
$$

Proof. Let $\psi \in \mathscr{D}$. Then, taking the limit $t \rightarrow \infty$ in (37) and using Lemma 8, we obtain

$$
\begin{aligned}
& \langle\psi, A \phi\rangle \\
& \quad=-i \int_{0}^{\infty} d s \int_{M} d \mu(k)\left\langle\psi, e^{i s\left(H-E_{0}(H)+u(k)\right)} S(k) \phi\right\rangle .
\end{aligned}
$$

For all $\varepsilon>0$, we have

$$
\begin{aligned}
& \left|e^{-\varepsilon s} \int_{M} d \mu(k)\left\langle\psi, e^{i s\left(H-E_{0}(H)+u(k)\right)} S(k) \phi\right\rangle\right| \\
& \quad \leq\left|\int_{M} d \mu(k)\left\langle\psi, e^{i s\left(H-E_{0}(H)+u(k)\right)} S(k) \phi\right\rangle\right| .
\end{aligned}
$$

By (c) in (A.2)(iii), the right hand side is integrable in $s \in[0, \infty)$. Hence, by the Lebesgue dominated convergence theorem, we have

$$
\begin{gathered}
\langle\psi, A \phi\rangle=-i \lim _{\varepsilon \rightarrow 0} \int_{0}^{\infty} d s e^{-\varepsilon s} \int_{M} d \mu(k) \\
\cdot\left\langle\psi, e^{i s\left(H-E_{0}(H)+u(k)\right)} S(k) \phi\right\rangle .
\end{gathered}
$$

Note that

$$
\begin{gathered}
\int_{0}^{\infty} d s e^{-\varepsilon s} \int_{M}\left|\left\langle\psi, e^{i s\left(H-E_{0}(H)+u(k)\right)} S(k) \phi\right\rangle\right| \\
\leq \int_{0}^{\infty} d s e^{-\varepsilon s} \int_{M}\|\psi\|\|S(k) \phi\| d \mu(k)<\infty .
\end{gathered}
$$

Hence, by Fubini's theorem, we have

$$
\begin{aligned}
\int_{0}^{\infty} d s e^{-\varepsilon s} \int_{M} d \mu(k)\left\langle\psi, e^{i s\left(H-E_{0}(H)+u(k)\right)} S(k) \phi\right\rangle \\
=\int_{M} d \mu(k) \\
\cdot\left\langle\psi, i\left(H-E_{0}(H)+u(k)+i \varepsilon\right)^{-1} S(k) \phi\right\rangle .
\end{aligned}
$$

Therefore

$$
\begin{aligned}
& \langle\psi, A \phi\rangle=\lim _{\varepsilon \rightarrow 0} \int_{M} d \mu(k) \\
& \cdot\left\langle\psi,\left(H-E_{0}(H)+u(k)+i \varepsilon\right)^{-1} S(k) \phi\right\rangle .
\end{aligned}
$$


It is easy to see that, for $\mu$-a.e. $k$,

$$
\begin{gathered}
\lim _{\varepsilon \rightarrow 0}\left\langle\psi,\left(H-E_{0}(H)+u(k)+i \varepsilon\right)^{-1} S(k) \phi\right\rangle \\
\quad=\left\langle\psi,\left(H-E_{0}(H)+u(k)\right)^{-1} S(k) \phi\right\rangle, \\
\left|\left\langle\psi,\left(H-E_{0}(H)+u(k)+i \varepsilon\right)^{-1} S(k) \phi\right\rangle\right| \\
\quad \leq\|\psi\| \frac{\|S(k) \phi\|}{u(k)} .
\end{gathered}
$$

Hence we can apply the Lebesgue dominated convergence theorem to obtain

$$
\begin{aligned}
& \langle\psi, A \phi\rangle \\
& =\left\langle\psi,\left(\int_{M}\left(H-E_{0}(H)+u(k)\right)^{-1} S(k) \phi d \mu(k)\right)\right\rangle .
\end{aligned}
$$

Thus (44) holds.

\section{Main Theorem}

We now state and prove the main theorem in the present paper. For this purpose, we first rewrite the theory in the preceding section in a form suitable for applications to models in quantum field theory.

We assume Hypothesis 1 . Let $(K, \Sigma, \mu)$ be a $\sigma$-finite measure space and let $\omega$ be a nonnegative $\Sigma$-measurable function on $K$ such that $0<\omega(k)<\infty$, $\mu$-a.e. $k \in K$. We set

$$
X:=L^{2}(K, d \mu)
$$

and, for each $s \in \mathbb{R}$, introduce a subspace

$$
X_{s}:=\left\{\left.f \in X\left|\int_{K} \omega(k)^{s}\right| f(k)\right|^{2} d \mu(k)<\infty\right\} .
$$

Suppose that there exists a family $\{A(f) \mid f \in X\}$ of densely defined closed linear operators on $\mathscr{H}$ such that, for all $f \in X, D\left(L^{1 / 2}\right) \subset D(A(f))$ and, for all $\psi \in D\left(L^{1 / 2}\right)$,

$$
\begin{aligned}
A(z f+w g) \psi=z^{*} A(f) \psi+w^{*} A(g) \psi, & \\
& f, g \in X, z, w \in \mathbb{C},
\end{aligned}
$$

where $z^{*}$ is the complex conjugate of $z \in \mathbb{C}$. We introduce an operator:

$$
A_{0}(f, t):=e^{-i t H_{0}} A(f) e^{i t H_{0}}, \quad t \in \mathbb{R}, f \in X .
$$

For the operator $A(f)$, we assume conditions similar to (A.1) and (A.2) for $A$ in the preceding section.

Hypothesis 2. (i) For some $\alpha \in(0,1]$ and $\beta \geq 0, D\left(H_{0}^{\alpha}\right) \subset$ $D(A(f)), f \in X_{-\beta}$ and for each $f \in X_{-\beta}$, there exist constants $a_{f}, b_{f} \geq 0$ such that

$$
\|A(f) \psi\| \leq a_{f}\left\|H_{0}^{\alpha} \psi\right\|+b_{f}\|\psi\|, \quad \psi \in D\left(H_{0}^{\alpha}\right) .
$$

(ii) There exists a core $\mathscr{D}$ of $H_{0}^{\alpha}$ such that

$$
\lim _{t \rightarrow \infty} A(f) e^{i t H_{0}} \psi=0, \quad \psi \in \mathscr{D}, f \in X_{-\beta} .
$$

(iii) There exists a dense subspace $Y \subset X_{-\beta}$ such that, for all $t \in \mathbb{R}$ and $f \in Y,\left(H_{0}, A_{0}(f, t)\right)$ has weak commutator on $D(H)$ and, for all $\psi \in D(H),\left[H_{0}, A_{0}(f, t)\right]_{\mathrm{w}}^{D(H)} \psi$ is strongly continuous in $t$.

(iv) For all $\psi \in D(H)$ and $f \in Y, A_{0}(f, t) \psi$ is strongly differentiable in $t$ and

$$
\frac{d A_{0}(f, t) \psi}{d t}=i\left[H_{0}, A_{0}(f, t)\right]_{\mathrm{w}}^{D(H)} \psi, \quad t \in \mathbb{R} .
$$

(v) For all $t \in \mathbb{R}, f \in Y,\left(H_{1}, A_{0}(f, t)\right)$ has weak commutator on $D(H)$ and, for all $\psi \in D(H),\left[H_{1}, A_{0}(f, t)\right]_{\mathrm{w}}^{D(H)} \psi$ is strongly continuous in $t$. Moreover, for $\mu$-a.e. $k \in K$, there exists a densely defined linear operator $T(k)$ on $\mathscr{H}$ with $D\left(T(k)^{*}\right) \cap D(T(k)) \supset D(H)$ such that the following hold:

(a) for all $\psi, \chi \in D(H)$ and $f \in Y, \int_{K} \mid f(k)\langle\chi$, $T(k) \psi\rangle \mid d \mu(k)<\infty$ and for all $t \in \mathbb{R}$,

$$
\begin{aligned}
\langle\chi & \left.,\left[H_{1}, A_{0}(f, t)\right]_{\mathrm{w}}^{D(H)} \psi\right\rangle \\
= & \int_{K} e^{i t \omega(k)} f(k)^{*}\langle\chi, T(k) \psi\rangle d \mu(k) ;
\end{aligned}
$$

(b) for all $\phi \in P_{H} \mathscr{H}$ and $f \in Y$,

$$
\begin{aligned}
& \int_{K}|f(k)|\|T(k) \phi\| d \mu(k)<\infty, \\
& \int_{K}|f(k)| \frac{\|T(k) \phi\|}{\omega(k)} d \mu(k)<\infty ;
\end{aligned}
$$

(c) there exists a dense subspace $Y_{0} \subset Y$ such that, for all $\psi \in \mathscr{D}, f \in Y_{0}$, and $\phi \in P_{H} \mathscr{H}$,

$$
\begin{aligned}
& \int_{0}^{\infty}\left|\int_{K} f(k)^{*}\left\langle\psi, e^{i s\left(H-E_{0}(H)+\omega(k)\right)} T(k) \phi\right\rangle d \mu(k)\right| d s \\
& \quad<\infty .
\end{aligned}
$$
$L$.

We need an assumption on a relation between $A(f)$ and

Hypothesis 3. There exist a complete orthonormal system (CONS) $\left\{e_{n}\right\}_{n=1}^{\infty}$ of $X$ with $e_{n} \in Y, n \in \mathbb{N}$, and a constant $\varepsilon_{0} \in[0,1)$ such that

$$
\sup _{\phi \in D\left(L^{1 / 2}\right) \cap P_{H} \mathscr{H},\|\phi\|=1}\left(\left\|L^{1 / 2} \phi\right\|^{2}-\sum_{n=1}^{\infty}\left\|A\left(e_{n}\right) \phi\right\|^{2}\right) \leq \varepsilon_{0} .
$$

The main theorem in the present paper is as follows. 
Theorem 12. Assume Hypotheses 1-3. Suppose that there exist nonnegative functions $f_{1}$ and $f_{2}$ on $(K, \Sigma)$ such that $f_{1}+$ $\omega^{-1} f_{2} \in X$ with

$$
\begin{aligned}
& \gamma:=\left\|f_{1}+\omega^{-1} f_{2}\right\|^{2}<1-\varepsilon_{0}, \\
&\left\|T(k)^{*} \psi\right\| \leq f_{1}(k)\left\|\left(H-E_{0}(H)+\omega(k)\right) \psi\right\| \\
&+f_{2}(k)\|\psi\|,
\end{aligned}
$$

Then,

$$
\psi \in D(H), \mu \text {-a.e. } k \in K \text {. }
$$

(i) any $\phi \in P_{H} \mathscr{H}$ with $\|\phi\|=1$ overlaps with $P_{H_{0}} \mathscr{H}$;

(ii)

$$
m_{L}(H) \leq \frac{1}{1-\gamma-\varepsilon_{0}} m\left(H_{0}\right)
$$

where $m_{L}(H)$ is defned by (9); moreover, if

$$
\gamma+\varepsilon_{0}<\frac{1}{m\left(H_{0}\right)+1}
$$

in addition, then

$$
m_{L}(H) \leq m\left(H_{0}\right) ;
$$

(iii) suppose that $P_{H} \mathscr{H} \subset D\left(L^{1 / 2}\right)$ in addition; then

$$
m(H) \leq \frac{1}{1-\gamma-\varepsilon_{0}} m\left(H_{0}\right) ;
$$

in particular, if (67) holds, then

$$
m(H) \leq m\left(H_{0}\right) ;
$$

(iv) if $P_{H} \mathscr{H} \subset D\left(L^{1 / 2}\right), m\left(H_{0}\right)=1$, and $\gamma+\varepsilon_{0}<1 / 2$, then $m(H)=1$.

To prove Theorem 12, we need some lemmas.

Lemma 13. Assume Hypothesis 2. Then, for all $\phi \in P_{H} \mathscr{H}$ and $f \in Y$,

$$
\begin{aligned}
& A(f) \phi \\
& \quad=\int_{K} f(k)^{*}\left(H-E_{0}(H)+\omega(k)\right)^{-1} T(k) \phi d \mu(k) .
\end{aligned}
$$

Proof. One can apply Theorem 11 with $A=A(f)$. Then (71) follows.

Lemma 14. Assume Hypothesis 2. Let $\phi \in P_{H} \mathscr{H}$ and

$$
\int_{K}\left\|\left(H-E_{0}(H)+\omega(k)\right)^{-1} T(k) \phi\right\|^{2} d \mu(k)<\infty .
$$

Then, for any CONS $\left\{e_{n}\right\}_{n=1}^{\infty}$ of $X$ with $e_{n} \in Y(n \in \mathbb{N})$,

$$
\begin{aligned}
\sum_{n=1}^{\infty} & \left\|A\left(e_{n}\right) \phi\right\|^{2} \\
& =\int_{K}\left\|\left(H-E_{0}(H)+\omega(k)\right)^{-1} T(k) \phi\right\|^{2} d \mu(k) .
\end{aligned}
$$

Proof. Define a mapping $T: X \rightarrow \mathscr{H}$ by

$$
\begin{array}{r}
T f:=\int_{K} f(k)\left(H-E_{0}(H)+\omega(k)\right)^{-1} T(k) \phi d \mu(k), \\
f \in X .
\end{array}
$$

Then, in quite the same way as in the proof of [12, Lemma 2.6], one can show that $T$ is Hilbert-Schmidt and

$$
\|T\|_{2}^{2}=\int_{K}\left\|\left(H-E_{0}(H)+\omega(k)\right)^{-1} T(k) \phi\right\|^{2} d \mu(k),
$$

where $\|T\|_{2}$ is the Hilbert-Schmidt norm of $T$. In general, for any CONS $\left\{f_{n}\right\}_{n=1}^{\infty}$ of $X,\|T\|_{2}^{2}=\sum_{n=1}^{\infty}\left\|T f_{n}\right\|^{2}$. The set $\left\{e_{n}^{*}\right\}_{n=1}^{\infty}$ also is a CONS of $X$. By (71), we have $T e_{n}^{*}=A\left(e_{n}\right) \phi$. Thus (73) holds.

We are now ready to prove Theorem 12 .

Proof of Theorem 12. Let $k \in K$ be such that $0<\omega(k)<\infty$. Then (65) implies that $T(k)^{*}\left(H-E_{0}(H)+\omega(k)\right)^{-1}$ is bounded with operator norm

$$
\left\|T(k)^{*}\left(H-E_{0}(H)+\omega(k)\right)^{-1}\right\| \leq f_{1}(k)+\frac{f_{2}(k)}{\omega(k)} .
$$

Hence $\left(H-E_{0}(H)+\omega(k)\right)^{-1} T(k)$ is bounded with domain $D(T(k))$ and

$$
\left\|\left(H-E_{0}(H)+\omega(k)\right)^{-1} T(k)\right\| \leq f_{1}(k)+\frac{f_{2}(k)}{\omega(k)} .
$$

Hence, for all $\psi \in D(H)$,

$$
\begin{aligned}
& \int_{K}\left\|\left(H-E_{0}(H)+\omega(k)\right)^{-1} T(k) \psi\right\|^{2} d \mu(k) \\
& \quad \leq \gamma\|\psi\|^{2} .
\end{aligned}
$$

Therefore, by Lemma 14 and Hypothesis 3, we have

$$
\left\|L^{1 / 2} \phi\right\|^{2} \leq \gamma+\varepsilon_{0}, \quad \phi \in D\left(L^{1 / 2}\right) \cap P_{H} \mathscr{H},\|\phi\|=1 .
$$

Thus, by Theorem 1, we obtain (66). The other parts of Theorem 12 easily follow from Theorem 1 and Corollaries 2 and 3 .

From the purely operator theoretical point of view, Theorem 12 can be regarded as a comparison theorem on the degeneracy of ground states in the framework given by the quintuple $\left(\mathscr{H}, H_{0}, H_{1}, L,\{A(f) \mid f \in X\}\right)$.

\section{Remarks for Applications}

As for applications of Theorem 12 to quantum field models, we describe only basic aspects, because full descriptions of applications need many pages and it may be suitable to present them in a separate paper.

The Hilbert space $\mathscr{H}$ in the abstract theory may have different concrete realizations depending on quantum field models. Here we present a unified treatment of various 
models in quantum field theory, taking as $\mathscr{H}$ a general Hilbert space. This will make less work in applications.

Let $W_{\mathrm{b}}$ and $W_{\mathrm{f}}$ be complex Hilbert spaces. Then the boson Fock space $\mathscr{F}_{\mathrm{b}}\left(W_{\mathrm{b}}\right)$ over $W_{\mathrm{b}}$ is defined by

$$
\begin{gathered}
\mathscr{F}_{\mathrm{b}}\left(W_{\mathrm{b}}\right):=\bigoplus_{n=0}^{\infty} \bigotimes_{\mathrm{s}}^{n} W_{\mathrm{b}}=\left\{\Psi=\left\{\Psi^{(n)}\right\}_{n=0}^{\infty} \mid \Psi^{(n)}\right. \\
\left.\in \bigotimes_{\mathrm{s}}^{n} W_{\mathrm{b}}, \quad n \geq 0,\|\Psi\|^{2}:=\sum_{n=0}^{\infty}\left\|\Psi^{(n)}\right\|^{2}<\infty\right\},
\end{gathered}
$$

where $\bigotimes_{\mathrm{s}}^{n} W_{\mathrm{b}}$ denotes the $n$-fold symmetric tensor product Hilbert space of $W_{\mathrm{b}}$ with convention $\bigotimes_{\mathrm{s}}^{0} W_{\mathrm{b}}:=\mathbb{C}$. On the other hand, the fermion Fock space $\mathscr{F}_{\mathrm{f}}\left(W_{\mathrm{f}}\right)$ over $W_{\mathrm{f}}$ is defined by

$$
\mathscr{F}_{\mathrm{f}}\left(W_{\mathrm{f}}\right):=\bigoplus_{n=0}^{\infty} \bigotimes_{\mathrm{as}}^{n} W_{\mathrm{f}},
$$

where $\bigotimes_{\mathrm{as}}^{n} W_{\mathrm{f}}$ is the $n$-fold antisymmetric tensor product Hilbert space of $W_{\mathrm{f}}$ with convention $\bigotimes_{\mathrm{as}}^{0} W_{\mathrm{f}}:=\mathbb{C}$. The bosonfermion Fock space $\mathscr{F}_{\mathrm{BF}}\left(W_{\mathrm{b}}, W_{\mathrm{f}}\right)$ over $\left(W_{\mathrm{b}}, W_{\mathrm{f}}\right)$ is defined by

$$
\mathscr{F}_{\mathrm{BF}}\left(W_{\mathrm{b}}, W_{\mathrm{f}}\right):=\mathscr{F}_{\mathrm{b}}\left(W_{\mathrm{b}}\right) \otimes \mathscr{F}_{\mathrm{f}}\left(W_{\mathrm{f}}\right) .
$$

This Hilbert space is a Hilbert space for a quantum system in which a Bose field interacts with a Fermi field.

A general Hilbert space unifying Hilbert spaces for various quantum field models is given by

$$
\mathscr{H}=\mathfrak{h} \otimes \mathscr{F}_{\mathrm{BF}}\left(W_{\mathrm{b}}, W_{\mathrm{f}}\right),
$$

where $\mathfrak{h}$ is a complex Hilbert space. Indeed, $\mathscr{H}$ includes, as special cases, three types of Hilbert spaces which appear typically in quantum field theory:

(i) In the case where $W_{\mathrm{f}}=\{0\}$, then $\mathscr{F}_{\mathrm{f}}\left(W_{\mathrm{f}}\right)=\mathbb{C}$. Hence, in this case, $\mathscr{H}$ is identified with $\mathfrak{h} \otimes \mathscr{F}_{\mathrm{b}}\left(W_{\mathrm{b}}\right)$, which is a Hilbert space for a general quantum system interacting with a Bose field. In particular, if $\mathfrak{h}=\mathbb{C}$, then $\mathscr{H}$ is identified with $\mathscr{F}_{\mathrm{b}}\left(W_{\mathrm{b}}\right)$.

(ii) In the case where $W_{\mathrm{b}}=\{0\}, \mathscr{H}$ is identified with $\mathfrak{h} \otimes \mathscr{F}_{\mathrm{f}}\left(W_{\mathrm{f}}\right)$, which is a Hilbert space for a general quantum system interacting with a Fermi field. In particular, if $\mathfrak{h}=\mathbb{C}$, then $\mathscr{H}$ is identified with $\mathscr{F}_{\mathrm{f}}\left(W_{\mathrm{f}}\right)$.

(iii) In the case where $\mathfrak{h}=\mathbb{C}, \mathscr{H}$ is identified with $\mathscr{F}_{\mathrm{BF}}\left(W_{\mathrm{b}}, W_{\mathrm{f}}\right)$.

In this sense, at least for applications to quantum field theory, the above choice of the Hilbert space $\mathscr{H}$ is general enough.

Let $A$ be a nonnegative self-adjoint operator on $\mathfrak{h}$ having ground state with $E_{0}(A)=0$ and $m(A)<\infty$. In what follows, \# denotes either b or $\mathrm{f}$. Let $S_{\#}$ be a nonnegative injective self-adjoint operator on $W_{\#}$ and denote by $d \Gamma_{\#}\left(S_{\#}\right)$ the second quantization of $S_{\#}$ on $\mathscr{F}_{\#}\left(W_{\#}\right)$. Let $H_{1}$ be a symmetric operator on $\mathscr{H}$. Then the following operator $H$ serves as unification of Hamiltonians of various quantum field models:

$$
H=H_{0}+H_{1}
$$

where

$$
H_{0}:=A \otimes I \otimes I+I \otimes d \Gamma_{\mathrm{b}}\left(S_{\mathrm{b}}\right) \otimes I+I \otimes I \otimes d \Gamma_{\mathrm{f}}\left(S_{\mathrm{f}}\right),
$$

and $H_{1}$ is not necessarily $H_{0}$-bounded.

Since $S_{\#}$ is nonnegative and injective, it follows that $d \Gamma_{\#}\left(S_{\#}\right)$ has a unique ground state with zero ground state energy and

$$
\begin{aligned}
& \operatorname{ker} d \Gamma_{\mathrm{b}}\left(S_{\mathrm{b}}\right)=\mathscr{F}_{\mathrm{b}}^{(0)}:=\left\{c \Omega_{\mathrm{b}} \mid c \in \mathbb{C}\right\}, \\
& \operatorname{ker} d \Gamma_{\mathrm{f}}\left(S_{\mathrm{f}}\right)=\mathscr{F}_{\mathrm{f}}^{(0)}:=\left\{c \Omega_{\mathrm{f}} \mid c \in \mathbb{C}\right\},
\end{aligned}
$$

where $\Omega_{\#}:=\{1,0,0, \ldots\}$ is the Fock vacuum in $\mathscr{F}_{\#}\left(W_{\#}\right)$. We denote by $P_{\#}$ the orthogonal projection onto $\mathscr{F}_{\#}^{(0)}$ from $\mathscr{F}_{\#}\left(W_{\#}\right)$.

The operator $H_{0}$ has ground state with $E_{0}\left(H_{0}\right)=0$ and

$$
P_{\mathrm{H}_{0}} \mathscr{H}=\operatorname{ker} A \otimes \mathscr{F}_{\mathrm{b}}^{(0)} \otimes \mathscr{F}_{\mathrm{f}}^{(0)} .
$$

We have

$$
P_{H_{0}}=P_{A} \otimes P_{\mathrm{b}} \otimes P_{\mathrm{f}}
$$

Hence

$$
m\left(H_{0}\right)=m(A) .
$$

We denote by $N_{\#}$ the number operator on $\mathscr{F}_{\#}\left(W_{\#}\right): N_{\#}:=$ $d \Gamma_{\#}(I)$. The operator

$$
Q_{A}:=I-P_{A}
$$

is the orthogonal projection onto $(\operatorname{ker} A)^{\perp}$ (the orthogonal complement of $\operatorname{ker} A$ ). For each $\varepsilon>0$, we define an operator $L_{\varepsilon}$ by

$$
\begin{aligned}
L_{\varepsilon}:= & (1+\varepsilon) I \otimes N_{\mathrm{b}} \otimes I+\left(1+\frac{1}{4 \varepsilon}\right) I \otimes I \otimes N_{\mathrm{f}}+Q_{A} \\
& \otimes P_{\mathrm{b}} \otimes P_{\mathrm{f} .}
\end{aligned}
$$

Lemma 15. For any $\varepsilon>0$,

$$
I \preceq L_{\varepsilon}+P_{H_{0}} .
$$

Proof. It is easy to see that

$$
\begin{aligned}
& I \preceq N_{\mathrm{b}}^{1 / 2}+P_{\mathrm{b}}, \\
& I \preceq N_{\mathrm{f}}^{1 / 2}+P_{\mathrm{f}} .
\end{aligned}
$$

Hence

$$
I \preceq I \otimes\left(N_{\mathrm{b}}^{1 / 2}+P_{\mathrm{b}}\right) \otimes\left(N_{\mathrm{f}}^{1 / 2}+P_{\mathrm{f}}\right) .
$$

By this inequality and the fact that $P_{\#} \leq I$ and $Q_{A}+P_{A}=I$, we obtain

$$
\begin{aligned}
I \preceq & I \otimes N_{\mathrm{b}}^{1 / 2} \otimes N_{\mathrm{f}}^{1 / 2}+I \otimes N_{\mathrm{b}}^{1 / 2} \otimes I+I \otimes I \otimes N_{\mathrm{f}}^{1 / 2} \\
& +Q_{\mathrm{A}} \otimes P_{\mathrm{b}} \otimes P_{\mathrm{f}}+P_{H_{0}} .
\end{aligned}
$$

By the functional calculus,

$$
I \otimes N_{\mathrm{b}}^{1 / 2} \otimes N_{\mathrm{f}}^{1 / 2} \preceq \varepsilon I \otimes N_{\mathrm{b}} \otimes I+\frac{1}{4 \varepsilon} I \otimes I \otimes N_{\mathrm{f}} .
$$

Since $\sigma\left(N_{\#}\right)=\{0\} \cup \mathbb{N}$, it follows that $N_{\#}^{1 / 2} \preceq N_{\#}$. Hence (92) is obtained. 
In the present framework, the operator $L_{\varepsilon}$ will be the operator $L$ in Hypothesis 1.

Remark 16. The parameter $\varepsilon>0$ in $L_{\varepsilon}$ is introduced to maintain the best possibility of (92) when the Hilbert space $\mathscr{H}$ is reduced to $\mathfrak{h} \otimes \mathscr{F}_{\mathrm{b}}\left(W_{\mathrm{b}}\right)$ (the case where $W_{\mathrm{f}}=\{0\}$ so that $N_{\mathrm{f}}=0$ and $P_{\mathrm{f}}=I$ ) or $\mathscr{F}_{\mathrm{f}}\left(W_{\mathrm{f}}\right)$ (the case where $W_{\mathrm{b}}=\{0\}$ so that $N_{\mathrm{b}}=0$ and $\left.P_{\mathrm{b}}=I\right)$.

We next describe a candidate for the operator $A(f)$ in Section 4. For this purpose, we use an isomorphism between a separable Hilbert space and an $L^{2}$ space. Hence we assume that $W_{\#}$ is separable. Then, by the multiplication operator form of the spectral theorem on a self-adjoint operator [8, Theorem VIII.4], there exist a finite measure space $\left(K_{\#}, \Sigma_{\#}, \mu_{\#}\right)$, a unitary operator $U_{\#}: W_{\#} \rightarrow L^{2}\left(K_{\#}, d \mu_{\#}\right)$, and a nonnegative function $\omega_{\#}$ on $K_{\#}$ satisfying $0<\omega_{\#}(k)<\infty$, $\mu_{\#}$-a.e. $k \in K_{\#}$ so that

$$
U S_{\#} U^{-1}=\omega_{\#} \text {, }
$$

where the right hand side denotes the multiplication operator by the function $\omega_{\#}$ on $L^{2}\left(K_{\#}, d \mu_{\#}\right)$. The isomorphism $U_{\#}$ induces the isomorphism

$$
\Gamma\left(U_{\#}\right): \mathscr{F}_{\#}\left(W_{\#}\right) \longrightarrow \mathscr{F}_{\#}\left(L^{2}\left(K_{\#}, d \mu_{\#}\right)\right)
$$

defined by

$$
\Gamma\left(U_{\#}\right):=\bigoplus_{n=0}^{\infty} \bigotimes^{n} U_{\#}
$$

with $\bigotimes^{0} U_{\#}:=1$.

In what follows we freely use the identification of $W_{\#}$ and $\mathscr{F}_{\#}\left(W_{\#}\right)$ with $L^{2}\left(K_{\#}, d \mu_{\#}\right)$ and $\mathscr{F}_{\#}\left(L^{2}\left(K_{\#}, d \mu_{\#}\right)\right)$, respectively.

Let

$$
K:=K_{\mathrm{b}} \cup K_{\mathrm{f}}
$$

with $K_{\mathrm{b}} \cap K_{\mathrm{f}}:=\emptyset$ and let $\mu$ be the measure on $K$ such that the restriction of $\mu$ to $K_{\#}$ is equal to $\mu_{\#}$. For each element $\left(f_{1}, f_{2}\right) \in$ $L^{2}\left(K_{\mathrm{b}}, d \mu_{\mathrm{b}}\right) \oplus L^{2}\left(K_{\mathrm{f}}, d \mu_{\mathrm{f}}\right)$, we define a function $f$ on $K$ by

$$
f(k):= \begin{cases}f_{1}(k), & \text { if } k \in K_{\mathrm{b}}, \\ f_{2}(k), & \text { if } k \in K_{\mathrm{f}} .\end{cases}
$$

Then it is easy to see that the correspondence $\left(f_{1}, f_{2}\right) \mapsto f$ gives an isomorphism between $L^{2}\left(K_{\mathrm{b}}, d \mu_{\mathrm{b}}\right) \oplus L^{2}\left(K_{\mathrm{f}}, d \mu_{\mathrm{f}}\right)$ and $L^{2}(K, d \mu)$. In this sense, we write $f=\left(f_{1}, f_{2}\right)$. Below we see that, in the present case, the Hilbert space $X$ in Section 4 is given by

$$
X:=L^{2}(K, d \mu) .
$$

We freely use the identification of $L^{2}\left(K_{\mathrm{b}}, d \mu_{\mathrm{b}}\right) \oplus L^{2}\left(K_{\mathrm{f}}, d \mu_{\mathrm{f}}\right)$ with $X$.

In the present case, we take $\omega$ in Section 4 as follows:

$$
\omega(k):= \begin{cases}\omega_{\mathrm{b}}(k), & \text { if } k \in K_{\mathrm{b}} \\ \omega_{\mathrm{f}}(k), & \text { if } k \in K_{\mathrm{f}}\end{cases}
$$

We are now ready to describe a candidate for $A(f)$ in Section 4. Let $u \in W_{\mathrm{b}}$ and let $a(u)$ be the boson annihilation operator on $\mathscr{F}_{\mathrm{b}}\left(W_{\mathrm{b}}\right)$, which is the densely defined closed linear operator on $\mathscr{F}_{\mathrm{b}}\left(W_{\mathrm{b}}\right)$ such that its adjoint $a(u)^{*}$ is of the form

$$
\begin{aligned}
\left(a(u)^{*} \Psi\right)^{(0)} & =0, \\
\left(a(u)^{*} \Psi\right)^{(n)} & =\sqrt{n} S_{n}\left(u \otimes \Psi^{(n-1)}\right), \\
& \Psi \in D\left(a(u)^{*}\right),
\end{aligned}
$$

where $S_{n}$ is the symmetrization operator on the $n$-fold tensor product $\otimes^{n} W_{\mathrm{b}}$ of $W_{\mathrm{b}}$. The following facts are well known:

(a.1) Canonical commutation relations: for all $u, u^{\prime} \in W_{\mathrm{b}}$,

$$
\begin{aligned}
{\left[a(u), a\left(u^{\prime}\right)^{*}\right] } & =\left\langle u, u^{\prime}\right\rangle, \\
{\left[a(u), a\left(u^{\prime}\right)\right] } & =0
\end{aligned}
$$

on the subspace

$$
\begin{aligned}
& \mathscr{F}_{\mathrm{b}, 0}\left(W_{\mathrm{b}}\right) \\
& :=\left\{\Psi \in \mathscr{F}_{\mathrm{b}}\left(W_{\mathrm{b}}\right) \mid \exists n_{0} \in \mathbb{N}, \forall n \geq n_{0}, \Psi^{(n)}=0\right\},
\end{aligned}
$$

where $[A, B]:=A B-B A$.

(a.2) For all $u \in W_{\mathrm{b}}, D\left(N_{\mathrm{b}}^{1 / 2}\right) \subset D(a(u))$ and $\|a(u) \Psi\| \leq$ $\|u\|\left\|N_{\mathrm{b}}^{1 / 2} \Psi\right\|, \Psi \in D\left(N_{\mathrm{b}}^{1 / 2}\right)$.

(a.3) For all CONS $\left\{u_{n}\right\}_{n=1}^{\infty}$ of $W_{\mathrm{b}}$,

$$
\left\|N_{\mathrm{b}}^{1 / 2} \Psi\right\|^{2}=\sum_{n=1}^{\infty}\left\|a\left(u_{n}\right) \Psi\right\|^{2}, \quad \Psi \in D\left(N_{\mathrm{b}}^{1 / 2}\right) .
$$

(a.4) For all $u \in D\left(S_{\mathrm{b}}^{-1 / 2}\right), D\left(d \Gamma_{\mathrm{b}}\left(S_{\mathrm{b}}\right)^{1 / 2}\right) \subset D(a(u))$ and

$$
\begin{aligned}
\|a(u) \Psi\| \leq\left\|S_{\mathrm{b}}^{-1 / 2} u\right\|\left\|d \Gamma_{\mathrm{b}}\left(S_{\mathrm{b}}\right)^{1 / 2} \Psi\right\|, & \\
\Psi & \in D\left(d \Gamma_{\mathrm{b}}\left(S_{\mathrm{b}}\right)^{1 / 2}\right) .
\end{aligned}
$$

(a.5) For all $t \in \mathbb{R}$ and $u \in W_{\mathrm{b}}, e^{i t d \Gamma_{\mathrm{b}}\left(S_{\mathrm{b}}\right)} a(u) e^{-i t d \Gamma_{\mathrm{b}}\left(S_{\mathrm{b}}\right)}=$ $a\left(e^{i t S_{\mathrm{b}}} u\right)$.

On the other hand, the fermion annihilation operator $b(v)$ $\left(v \in W_{\mathrm{f}}\right)$ on $\mathscr{F}_{\mathrm{f}}\left(W_{\mathrm{f}}\right)$ is the everywhere defined bounded linear operator on $\mathscr{F}_{\mathrm{f}}\left(W_{\mathrm{f}}\right)$ such that

$$
\begin{aligned}
& \left(b(v)^{*} \Psi\right)^{(0)}=0, \\
& \left(b(v)^{*} \Psi\right)^{(n)}=\sqrt{n} A_{n}\left(v \otimes \Psi^{(n-1)}\right),
\end{aligned}
$$

$$
\Psi \in \mathscr{F}_{\mathrm{f}}\left(W_{\mathrm{f}}\right),
$$

where $A_{n}$ is the antisymmetrization operator on $\otimes^{n} W_{\mathrm{f}}$. The following facts are well known: 
(b.1) Canonical anticommutation relations: for all $v, v^{\prime} \epsilon$ $W_{\mathrm{f}}$,

$$
\begin{gathered}
\left\{b(v), b\left(v^{\prime}\right)^{*}\right\}=\left\langle v, v^{\prime}\right\rangle, \\
\left\{b(v), b\left(v^{\prime}\right)\right\}=0,
\end{gathered}
$$

where $\{A, B\}:=A B+B A$.

(b.2) $\|b(v)\|=\left\|b(v)^{*}\right\|=\|v\|, v \in W_{\mathrm{f}}$.

(b.3) For all CONS $\left\{v_{n}\right\}_{n=1}^{\infty}$ of $W_{\mathrm{f}}$,

$$
\left\|N_{\mathrm{f}}^{1 / 2} \Psi\right\|^{2}=\sum_{n=1}^{\infty}\left\|b\left(v_{n}\right) \Psi\right\|^{2}, \quad \Psi \in D\left(N_{\mathrm{f}}^{1 / 2}\right) .
$$

(b.4) For all $t \in \mathbb{R}$ and $v \in W_{\mathrm{f}}, e^{i t d \Gamma_{\mathrm{f}}\left(S_{\mathrm{f}}\right)} b(v) e^{-i t d \Gamma_{\mathrm{f}}\left(S_{\mathrm{f}}\right)}=$ $b\left(e^{i t S_{\mathrm{f}}} v\right)$.

In the present case, we see that a candidate for $A(f)$ in Section 4 may be the closure of the following operator:

$$
\begin{gathered}
A_{\varepsilon}(f):=\sqrt{1+\varepsilon} I \otimes a\left(f_{1}\right) \otimes I+\sqrt{1+\frac{1}{4 \varepsilon}} I \otimes I \\
\otimes b\left(f_{2}\right), \quad f=\left(f_{1}, f_{2}\right) \in X,
\end{gathered}
$$

where $\varepsilon>0$ is arbitrary. We denote the closure of $A_{\varepsilon}(f)$ by the same symbol. The parameter $\varepsilon$ in $A_{\varepsilon}$ is introduced in correspondence to $L_{\varepsilon}$ defined by (91). It is easy to see that

$$
D\left(L_{\varepsilon}^{1 / 2}\right)=D\left(I \otimes N_{\mathrm{b}}^{1 / 2} \otimes I\right) \cap D\left(I \otimes I \otimes N_{\mathrm{f}}^{1 / 2}\right) .
$$

Hence $D\left(L_{\varepsilon}^{1 / 2}\right) \subset D\left(A_{\varepsilon}(f)\right)$ for all $f \in X$ and (55) holds.

It follows from (a.4) that, for all $f=\left(f_{1}, f_{2}\right) \in D\left(S_{\mathrm{b}}^{-1 / 2}\right) \oplus$ $W_{\mathrm{f}}, D\left(H_{0}^{1 / 2}\right) \subset D\left(A_{\varepsilon}(f)\right)$ and

$$
\begin{aligned}
& \left\|A_{\varepsilon}(f) \Psi\right\| \\
& \leq \quad \sqrt{1+\varepsilon}\left\|\left(I \otimes a\left(f_{1}\right) \otimes I\right) \Psi\right\| \\
& \quad+\sqrt{1+\frac{1}{4 \varepsilon}}\left\|f_{2}\right\|\|\Psi\| \\
& \leq \sqrt{1+\varepsilon}\left\|S_{\mathrm{b}}^{-1 / 2} f_{1}\right\|\left\|I \otimes d \Gamma_{\mathrm{b}}\left(S_{\mathrm{b}}\right)^{1 / 2} \otimes I \Psi\right\| \\
& \quad+\sqrt{1+\frac{1}{4 \varepsilon}}\left\|f_{2}\right\|\|\Psi\| \\
& \leq \sqrt{1+\varepsilon}\left\|S_{\mathrm{b}}^{-1 / 2} f_{1}\right\|\left\|H_{0}^{1 / 2} \Psi\right\|+\sqrt{1+\frac{1}{4 \varepsilon}}\left\|f_{2}\right\|\|\Psi\| .
\end{aligned}
$$

Hence Hypothesis 2(i) holds with $\beta=1$ and $\alpha=1 / 2$.

In the present case, we have

$$
\begin{aligned}
& A_{0}(f, t)=A_{\varepsilon}\left(e^{-i t \omega_{\mathrm{b}}} f_{1}, e^{-i t \omega_{\mathrm{f}}} f_{2}\right), \\
& \quad f=\left(f_{1}, f_{2}\right) \in X, t \in \mathbb{R},
\end{aligned}
$$

where, for notational simplicity, the dependence of $A_{0}(f, t)$ on $\varepsilon$ is not explicitly written.

To ensure that Hypothesis 2(ii) holds, we consider an additional condition:
(S) The self-adjoint operators $S_{\mathrm{b}}$ and $S_{\mathrm{f}}$ are purely absolutely continuous.

For a subspace $D_{\#}$ of $W_{\#}$, we define $\mathscr{F}_{\#, \text { fin }}(D) \subset \mathscr{F}_{\#}\left(W_{\#}\right)$ by

$$
\begin{aligned}
& \mathscr{F}_{\#, \text { fin }}\left(D_{\#}\right):=\operatorname{span}\left\{\Omega_{\#}, c_{\#}\left(g_{1}\right)^{*} \cdots c_{\#}\left(g_{n}\right)^{*} \Omega_{\#} \mid n\right. \\
& \left.\in \mathbb{N}, g_{j} \in D_{\#}, j=1, \ldots, n\right\},
\end{aligned}
$$

where, for a subset $V$ of a vector space, $\operatorname{span} V$ denotes the subspace algebraically spanned by all the vectors in $V$ and $c_{\mathrm{b}}(\cdot)=a(\cdot), c_{\mathrm{f}}(\cdot)=b(\cdot)$. For a subspace $\mathscr{G}_{\#}$ of $\mathscr{F}_{\#}\left(W_{\#}\right)$, we denote by $\mathfrak{h} \widehat{\otimes} \mathscr{G}_{\mathrm{b}} \widehat{\otimes} \mathscr{G}_{\mathrm{f}}$ the algebraic tensor product of $\mathfrak{h}, \mathscr{G}_{\mathrm{b}}$, and $\mathscr{G}_{\mathrm{f}}$.

Lemma 17. Assume (S). Then, for all $\Psi \in \mathfrak{h} \widehat{\otimes} \mathscr{F}_{\mathrm{b}, \text { fin }}\left(W_{\mathrm{b}}\right) \widehat{\otimes}$ $\mathscr{F}_{\text {f,fin }}\left(W_{\mathrm{f}}\right)$,

$$
\lim _{t \rightarrow \pm \infty} A_{\varepsilon}(f) e^{i t H_{0}} \Psi=0 .
$$

Proof. It is sufficient to prove the assertion for vectors $\Psi$ of the form

$$
\begin{aligned}
& \Psi=\psi \otimes a\left(u_{1}\right)^{*} \cdots a\left(u_{n}\right)^{*} \Omega_{\mathrm{b}} \otimes b\left(v_{1}\right)^{*} \cdots b\left(v_{m}\right)^{*} \Omega_{\mathrm{f}}, \\
& \psi \in \mathfrak{h}, u_{j} \in W_{\mathrm{b}}, \quad v_{\ell} \in W_{\mathrm{f}}, j=1, \ldots, n, \ell=1, \ldots, m, n, m \in \mathbb{N} .
\end{aligned}
$$

Let $f=\left(f_{1}, f_{2}\right) \in X$. Then, using (115), (a.1), and (b.1), we have

$$
\begin{aligned}
e^{-i t H_{0}} A_{\varepsilon}(f) e^{i t H_{0}} \Psi= & \sum_{k=1}^{n}\left\langle e^{-i t S_{\mathrm{b}}} f_{1}, u_{k}\right\rangle \Psi_{\mathrm{b}, k} \\
& +\sum_{\ell=1}^{m}\left\langle e^{-i t S_{\mathrm{f}}} f_{2}, v_{\ell}\right\rangle \Psi_{\mathrm{f}, \ell},
\end{aligned}
$$

where

$$
\begin{aligned}
\Psi_{\mathrm{b}, k} & \\
:= & \\
& \otimes a\left(u_{1}\right)^{*} \cdots a\left(u_{k-1}\right)^{*} a\left(u_{k+1}\right)^{*} \cdots a\left(u_{n}\right)^{*} \Omega_{\mathrm{b}} \\
& \otimes b\left(v_{1}\right)^{*} \cdots b\left(v_{m}\right)^{*} \Omega_{\mathrm{f}}, \\
\Psi_{\mathrm{f}, \ell} & :=(-1)^{\ell-1} \psi \otimes a\left(u_{1}\right)^{*} \cdots a\left(u_{n}\right)^{*} \Omega_{\mathrm{b}} \\
& \otimes b\left(v_{1}\right)^{*} \cdots b\left(v_{\ell-1}\right)^{*} b\left(v_{\ell+1}\right)^{*} \cdots b\left(v_{m}\right)^{*} \Omega_{\mathrm{f} .}
\end{aligned}
$$

By assumption (S), $\lim _{t \rightarrow \pm \infty}\left\langle e^{-i t S_{\mathrm{b}}} f_{1}, u_{k}\right\rangle=$ 0 and $\lim _{t \rightarrow \pm \infty}\left\langle e^{-i t S_{\mathrm{f}}} f_{2}, v_{\ell}\right\rangle=0$. Hence $\lim _{t \rightarrow \pm \infty} e^{-i t H_{0}} A_{\varepsilon}(f) e^{i t H_{0}} \Psi=0$. Since $e^{-i t H_{0}}$ is unitary, (117) follows.

Since $D(A) \widehat{\otimes} \mathscr{F}_{\mathrm{b} \text {,fin }}\left(D\left(S_{\mathrm{b}}\right)\right) \widehat{\otimes} \mathscr{F}_{\mathrm{f} \text {,fin }}\left(D\left(S_{\mathrm{f}}\right)\right)$ is a core of $H_{0}^{1 / 2}$, Lemma 17 implies that Hypothesis 2(ii) holds in the present case. 
It is well known that, for all $u \in D\left(S_{\mathrm{b}}\right) \cap D\left(S_{\mathrm{b}}^{-1 / 2}\right)$, $\left(d \Gamma_{\mathrm{b}}\left(S_{\mathrm{b}}\right), a(u)\right)$ has weak commutator on $D\left(d \Gamma_{\mathrm{b}}\left(S_{\mathrm{b}}\right)\right)$ and

$$
\begin{aligned}
{\left[d \Gamma_{\mathrm{b}}\left(S_{\mathrm{b}}\right), a(u)\right]_{\mathrm{w}}^{D\left(d \Gamma_{\mathrm{b}}\left(S_{\mathrm{b}}\right)\right)}=-a\left(S_{\mathrm{b}} u\right) } & \\
& \text { on } D\left(d \Gamma_{\mathrm{b}}\left(S_{\mathrm{b}}\right)\right) .
\end{aligned}
$$

Also, for all $v \in D\left(S_{\mathrm{f}}\right),\left(d \Gamma_{\mathrm{f}}\left(S_{\mathrm{f}}\right), b(v)\right)$ has weak commutator on $D\left(d \Gamma_{\mathrm{f}}\left(S_{\mathrm{f}}\right)\right)$ and

$$
\left[d \Gamma_{\mathrm{f}}\left(S_{\mathrm{f}}\right), b(v)\right]_{\mathrm{w}}^{D\left(d \Gamma_{\mathrm{f}}\left(S_{\mathrm{f}}\right)\right)}=-b\left(S_{\mathrm{f}} v\right) .
$$

Hence, by (115), $\left(H_{0}, A_{0}(f, t)\right)$ has weak commutator on $D\left(H_{0}\right)$ and

$$
\begin{array}{r}
{\left[H_{0}, A_{0}(f, t)\right]_{\mathrm{w}}^{D\left(H_{0}\right)}=-A_{\varepsilon}\left(e^{-i t S_{\mathrm{b}}} S_{\mathrm{b}} f_{1}, e^{-i t S_{\mathrm{f}}} S_{\mathrm{f}} f_{2}\right)} \\
\text { on } D\left(H_{0}\right) .
\end{array}
$$

Lemma 18. Let $f=\left(f_{1}, f_{2}\right) \in X$ with $f_{1} \in D\left(S_{\mathrm{b}}\right) \cap D\left(S_{\mathrm{b}}^{-1 / 2}\right)$ and $f_{2} \in D\left(S_{\mathrm{f}}\right)$. Then, for all $\Psi \in D\left(H_{0}\right),\left[H_{0}, A_{0}(f, t)\right]_{\mathrm{w}}^{D\left(H_{0}\right)} \Psi$ is strongly continuous in $t \in \mathbb{R}$.

Proof. For notational simplicity, we omit identity $I$ in tensor products of operators (e.g., $I \otimes a(u) \otimes I\left(u \in W_{\mathrm{b}}\right)$ is simply written as $a(u))$. For all $t, t^{\prime} \in \mathbb{R}$ and $\Psi \in D\left(H_{0}^{1 / 2}\right)$, we have by (a.4)

$$
\begin{aligned}
& \left\|a\left(e^{-i t^{\prime} S_{\mathrm{b}}} S_{\mathrm{b}} f_{1}\right) \Psi-a\left(e^{-i t S_{\mathrm{b}}} S_{\mathrm{b}} f_{1}\right) \Psi\right\| \\
& \leq\left\|e^{-i t^{\prime} \mathrm{b}_{\mathrm{b}}} S_{\mathrm{b}}^{1 / 2} f_{1}-e^{-i t S_{\mathrm{b}}} S_{\mathrm{b}}^{1 / 2} f_{1}\right\|\left\|H_{0}^{1 / 2} \Psi\right\| \\
& \longrightarrow 0 \\
& \left(t^{\prime} \longrightarrow t\right) .
\end{aligned}
$$

Similarly one can show that $\lim _{t^{\prime} \rightarrow t} b\left(e^{-i t^{\prime} S_{\mathrm{f}}} S_{\mathrm{f}} f_{2}\right) \Psi=$ $b\left(e^{-i t S_{\mathrm{f}}} S_{\mathrm{f}} f_{2}\right) \Psi$. Hence $A_{\varepsilon}\left(e^{-i t S_{\mathrm{b}}} S_{\mathrm{b}} f_{1}, e^{-i t S_{\mathrm{f}}} S_{\mathrm{f}} f_{2}\right) \Psi$ is strongly continuous in $t$. By this fact and (123), we obtain the desired result.

Lemma 19. For all $\Psi \in D\left(H_{0}\right)$ and $f \in X_{-1} \cap X_{2}, A_{0}(f, t) \Psi$ is strongly differentiable in $t$ and

$$
\frac{d A_{0}(f, t) \Psi}{d t}=i\left[H_{0}, A_{0}(f, t)\right]_{\mathrm{w}}^{D\left(H_{0}\right)} \Psi, \quad t \in \mathbb{R} .
$$

Proof. This follows from (115) and (123).

As for the weak commutator of $\left(H_{1}, A_{0}(f, t)\right)$, the following form gives unification of some models:

$$
\begin{aligned}
& {\left[H_{1}, A_{0}(f, t)\right]_{\mathrm{w}}^{D(H)} \Psi} \\
& \quad=\int_{K} e^{i t \omega(k)} f(k)^{*} T(k) \Psi d \mu(k), \\
& \Psi \in D(H), f \in\left[D\left(\omega_{\mathrm{b}}\right) \cap D\left(\omega_{\mathrm{b}}^{-1 / 2}\right)\right] \oplus D\left(\omega_{\mathrm{f}}\right),
\end{aligned}
$$

where $T(k)$ is a densely defined linear operator on $\mathscr{H}$ defined for $\mu$-a.e. $k \in K$, satisfying $D(T(k)) \cap D\left(T(k)^{*}\right) \supset D(H)$ and

$$
\begin{aligned}
& \int_{K}|f(k)|\|T(k) \Psi\| d \mu(k)<\infty, \\
& \int_{K} \frac{|f(k)|}{\omega(k)}\|T(k) \Psi\| d \mu(k)<\infty .
\end{aligned}
$$

Note that one can write

$$
\begin{aligned}
& {\left[H_{1}, A_{0}(f, t)\right]_{\mathrm{w}}^{D(H)} \Psi} \\
& =\int_{K_{\mathrm{b}}} e^{i t \omega_{\mathrm{b}}(k)} f_{1}(k)^{*} T_{1}(k) \Psi d \mu_{\mathrm{b}}(k) \\
& \quad+\int_{K_{\mathrm{f}}} e^{i t \omega_{\mathrm{f}}(k)} f_{2}(k)^{*} T_{2}(k) \Psi d \mu_{\mathrm{f}}(k),
\end{aligned}
$$

where $T_{1}(k):=T(k), k \in K_{\mathrm{b}}$ and $T_{2}(k):=T(k), k \in K_{\mathrm{f}}$.

To give an example of $H_{1}$ which has the abovementioned properties and is not relatively bounded with respect to $H_{0}$, we recall a basic object in $\mathscr{F}_{\mathrm{b}}\left(W_{\mathrm{b}}\right)$

$$
\phi(u):=\frac{1}{\sqrt{2}}\left(a(u)+a(u)^{*}\right), \quad u \in W_{\mathrm{b}},
$$

which is called the Segal field operator with test vector $u[9$, $\S X .7]$.

Example 20. Consider the case where $W_{\mathrm{b}}=L^{2}\left(\mathbb{R}^{d}\right)(d \in \mathbb{N})$ and $W_{\mathrm{f}}=\{0\}$ so that $\mathscr{H}=\mathfrak{h} \otimes \mathscr{F}_{\mathrm{b}}\left(L^{2}\left(\mathbb{R}^{d}\right)\right)$. In this case, $H_{0}$ becomes

$$
H_{0}=A \otimes I+I \otimes d \Gamma_{\mathrm{b}}\left(\omega_{\mathrm{b}}\right)
$$

and $A_{\varepsilon}(f)$ takes the form

$$
A(f):=I \otimes a(f), \quad f \in L^{2}\left(\mathbb{R}^{d}\right),
$$

where we set $\varepsilon=0$. Let $F$ be a nonnegative bounded continuous function on $\mathbb{R}$ and $\lambda \in L^{2}\left(\mathbb{R}^{d}\right) \backslash\{0\}$ such that $\lambda / \sqrt{\omega_{\mathrm{b}}} \in L^{2}\left(\mathbb{R}^{d}\right)$. Let $n \geq 1$ and

$$
H_{1}:=F(A) \otimes \phi(\lambda)^{2 n} .
$$

Then $H_{1}$ is a symmetric operator. It is proved that $H$ is selfadjoint (this is nontrivial and will be discussed elsewhere) and bounded below (this is trivial). If $n \geq 2$, then $H_{1}$ is not $H_{0}$ bounded.

One can show that $\left(H_{1}, A_{0}(f, t)\right)$ has weak commutator on $D(H)$ with

$$
\begin{aligned}
{\left[H_{1}, A_{0}(f, t)\right]_{\mathrm{w}}^{D(H)}=} & -\sqrt{2} n\left\langle e^{-i t \omega_{\mathrm{b}}} f, \lambda\right\rangle F(A) \\
& \otimes \phi(\lambda)^{2 n-1}
\end{aligned}
$$

on $D(H)$. Hence, in the present example, we have

$$
T(k)=-\sqrt{2} n \lambda(k) F(A) \otimes \phi(\lambda)^{2 n-1} .
$$

Under additional conditions, one can show that Hypothesis 2(v) and (65) hold (these facts also will be discussed elsewhere). 
Example 21. Consider the case where $\mathfrak{h}=\mathbb{C}$ and $W_{\mathrm{b}}=W_{\mathrm{f}}=$ $L^{2}\left(\mathbb{R}^{d}\right)(d \in \mathbb{N})$ so that $\mathscr{H}=\mathscr{F}_{\mathrm{b}}\left(L^{2}\left(\mathbb{R}^{d}\right)\right) \otimes \mathscr{F}_{\mathrm{f}}\left(L^{2}\left(\mathbb{R}^{d}\right)\right)$. We set $K_{\mathrm{b}}=\mathbb{R}^{d}, K_{\mathrm{f}}=\mathbb{R}^{d}$, and $K=K_{\mathrm{b}} \cup K_{\mathrm{f}}$. We denote by $\mu$ the measure on $K$ such that the restriction of $\mu$ to $K_{\#}$ is the $d$-dimensional Lebesgue measure. In this case, $H_{0}$ becomes

$$
\begin{gathered}
H_{0}=d \Gamma_{\mathrm{b}}\left(\omega_{\mathrm{b}}\right) \otimes I+I \otimes d \Gamma_{\mathrm{f}}\left(\omega_{\mathrm{f}}\right), \\
A_{\varepsilon}(f)=\sqrt{1+\varepsilon} a\left(f_{1}\right) \otimes I+\sqrt{1+\frac{1}{4 \varepsilon}} I \otimes b\left(f_{2}\right), \\
f=\left(f_{1}, f_{2}\right) \in L^{2}\left(\mathbb{R}^{d}\right) \otimes L^{2}\left(\mathbb{R}^{d}\right) .
\end{gathered}
$$

Let $\lambda \in L^{2}\left(\mathbb{R}^{d}\right)$ be as in Example 20 and let $v, w \in L^{2}\left(\mathbb{R}^{d}\right)$ be fixed and

$$
B:=b(v)^{*}+b(w) .
$$

Then, for $n \geq 2$, we define

$$
H_{1}:=\phi(\lambda) \otimes B^{*} B+\phi(\lambda)^{2 n} \otimes I .
$$

The operator $H_{1}$ is not $H_{0}$-bounded. It is easy to see that $H$ is bounded below (the self-adjointness of $H$ will be shown elsewhere). One can show that $\left(H_{1}, A_{0}(f, t)\right)$ has weak commutator on $D(H)$ and

$$
\begin{aligned}
& {\left[H_{1}, A_{0}(f, t)\right]_{\mathrm{w}}^{D(H)}=-\sqrt{\frac{1+\varepsilon}{2}}\left\langle e^{-i t \omega_{\mathrm{b}}} f_{1}, \lambda\right\rangle I \otimes B^{*} B} \\
& \quad-\sqrt{2(1+\varepsilon)} n\left\langle e^{-i t \omega_{\mathrm{b}}} f_{1}, \lambda\right\rangle \phi(\lambda)^{2 n-1} \otimes I \\
& \quad+\sqrt{1+\frac{1}{4 \varepsilon}}\left(\left\langle e^{-i t \omega_{\mathrm{f}}} f_{2}, v\right\rangle \phi(\lambda) \otimes B^{*}\right. \\
& \left.\quad-\left\langle e^{-i t \omega_{\mathrm{f}}} f_{2}, w\right\rangle \phi(\lambda) \otimes B\right) .
\end{aligned}
$$

Hence, in the present example, we have

$$
T(k)= \begin{cases}-\sqrt{\frac{1+\varepsilon}{2}} \lambda(k) I \otimes B^{*} B-\sqrt{2(1+\varepsilon)} n \lambda(k) \phi(\lambda)^{2 n-1} \otimes I, & \text { if } k \in K_{\mathrm{b}}, \\ \sqrt{1+\frac{1}{4 \varepsilon}}\left(v(k) \phi(\lambda) \otimes B^{*}-w(k) \phi(\lambda) \otimes B\right), & \text { if } k \in K_{\mathrm{f}} .\end{cases}
$$

Let $\left\{u_{n}\right\}_{n=1}^{\infty}$ (resp., $\left\{v_{n}\right\}_{n=1}^{\infty}$ ) be a CONS of $W_{\mathrm{b}}$ (resp., $W_{\mathrm{f}}$ ) and define $e_{n} \in W_{\mathrm{b}} \oplus W_{\mathrm{f}}$ as follows:

$$
\begin{aligned}
& e_{2 n-1}:=\left(u_{n}, 0\right), \\
& e_{2 n}:=\left(0, v_{n}\right), \\
& n \in \mathbb{N} .
\end{aligned}
$$

Then $\left\{e_{n}\right\}_{n=1}^{\infty}$ is a CONS of $W_{\mathrm{b}} \oplus W_{\mathrm{f} \cdot}$. By (a.3) and (b.3), we have

$$
\begin{aligned}
\sum_{n=1}^{\infty}\left\|A_{\varepsilon}\left(e_{n}\right) \Psi\right\|^{2}= & (1+\varepsilon)\left\|\left(I \otimes N_{\mathrm{b}}^{1 / 2} \otimes I\right) \Psi\right\|^{2} \\
& +\left(1+\frac{1}{4 \varepsilon}\right)\left\|I \otimes I \otimes N_{\mathrm{f}}^{1 / 2} \Psi\right\|^{2}
\end{aligned}
$$

for all $\Psi \in D\left(I \otimes N_{\mathrm{b}}^{1 / 2} \otimes I\right) \cap D\left(I \otimes I \otimes N_{\mathrm{f}}^{1 / 2}\right)$. Hence, by (91), we obtain

$$
\begin{gathered}
\sup _{\phi \in D\left(L_{\varepsilon}^{1 / 2}\right) \cap P_{H} \mathscr{H},\|\phi\|=1}\left(\left\|L_{\varepsilon}^{1 / 2} \phi\right\|^{2}-\sum_{n=1}^{\infty}\left\|A_{\varepsilon}\left(e_{n}\right) \phi\right\|^{2}\right) \\
=\sup _{\phi \in D\left(L_{\varepsilon}^{1 / 2}\right) \cap P_{H} \mathscr{H},\|\phi\|=1}\left\|Q_{A} \otimes P_{\mathrm{b}} \otimes P_{\mathrm{f}} \phi\right\|^{2} .
\end{gathered}
$$

Therefore we need only to show that there exists a constant $\varepsilon_{0} \in[0,1)$ such that

$$
\sup _{\phi \in D\left(L_{\varepsilon}^{1 / 2}\right) \cap P_{H} \mathscr{H},\|\phi\|=1}\left\|Q_{A} \otimes P_{\mathrm{b}} \otimes P_{\mathrm{f}} \phi\right\|^{2} \leq \varepsilon_{0} .
$$

Then Hypothesis 3 is satisfied. Estimate (143) can be obtained by extending the methods in $[6,7,10]$ to the present case.

In this way, for quantum field models within the class under consideration, one can obtain results (i)-(iii) in Theorem 12 (under additional conditions). The details will be given in a separate paper.

\section{Conflict of Interests}

The authors declare that there is no conflict of interests regarding the publication of this paper.

\section{Acknowledgment}

This work is supported by JSPS KAKENHI 15K04888.

\section{References}

[1] M. Reed and B. Simon, Methods of Modern Mathematical Physics IV: Analysis of Operators, Academic Press, New York, NY, USA, 1978.

[2] W. G. Faris, "Invariant cones and uniqueness of the ground state for fermion systems," Journal of Mathematical Physics, vol. 13, pp. 1285-1290, 1972.

[3] T. Miyao, "Nondegeneracy of ground states in nonrelativistic quantum field theory," Journal of Operator Theory, vol. 64, no. 1, pp. 207-241, 2010.

[4] T. Miyao, "Self-dual cone analysis in condensed matter physics," Reviews in Mathematical Physics, vol. 23, no. 7, pp. 749-822, 2011. 
[5] F. Hiroshima, "Perturbations of embedded eigenvalues in quantum field theory," Sûgaku, vol. 57, pp. 70-92, 2005 (Japanese), English translation: Sugaku Expositions, vol. 21, pp. 177-207, 2008.

[6] V. Bach, J. Fröhlich, and I. M. Sigal, "Quantum electrodynamics of confined nonrelativistic particles," Advances in Mathematics, vol. 137, no. 2, pp. 299-395, 1998.

[7] F. Hiroshima, "Multiplicity of ground states in quantum field models: applications of asymptotic fields," Journal of Functional Analysis, vol. 224, no. 2, pp. 431-470, 2005.

[8] M. Reed and B. Simon, Methods of Modern Mathematical Physics I: Functional Analysis, Academic Press, New York, NY, USA, 1972.

[9] M. Reed and B. Simon, Methods of Modern Mathematical Physics II: Fourier Analysis, Self-Adjointness, Academic Press, New York, NY, USA, 1975.

[10] A. Arai and M. Hirokawa, "On the existence and uniqueness of ground states of a generalized spin-boson model," Journal of Functional Analysis, vol. 151, no. 2, pp. 455-503, 1997.

[11] A. Arai, M. Hirokawa, and F. Hiroshima, "On the absence of eigenvectors of Hamilto-nians in a class of massless quantum field models without infrared cutoff,' Journal of Functional Analysis, vol. 168, no. 2, pp. 470-497, 1999.

[12] A. Arai, M. Hirokawa, and F. Hiroshima, "Regularities of ground states of quantum field models," Kyushu Journal of Mathematics, vol. 61, no. 2, pp. 321-372, 2007.

[13] A. Arai, "An abstract sum formula and its applications to special functions," Journal of Mathematical Analysis and Applications, vol. 167, no. 1, pp. 245-265, 1992. 


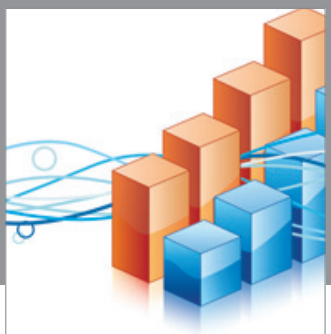

Advances in

Operations Research

vatem alat4

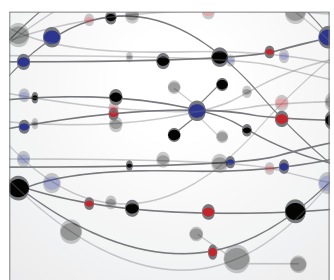

\section{The Scientific} World Journal
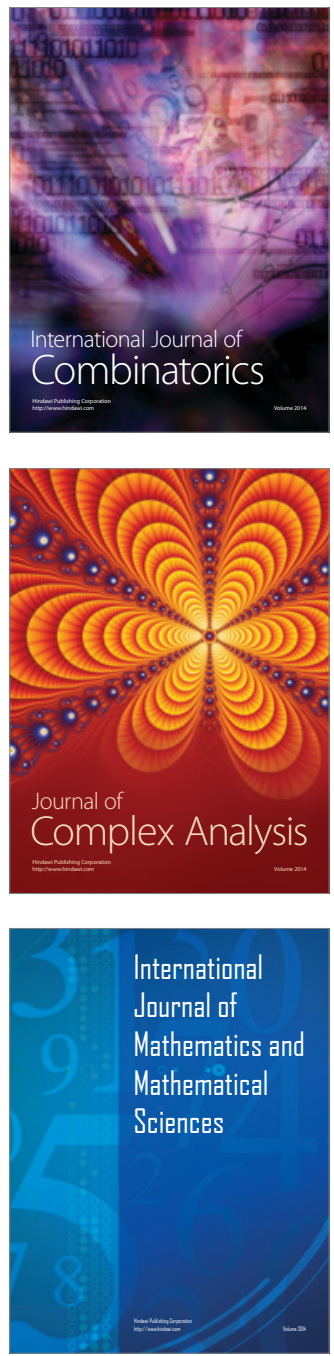
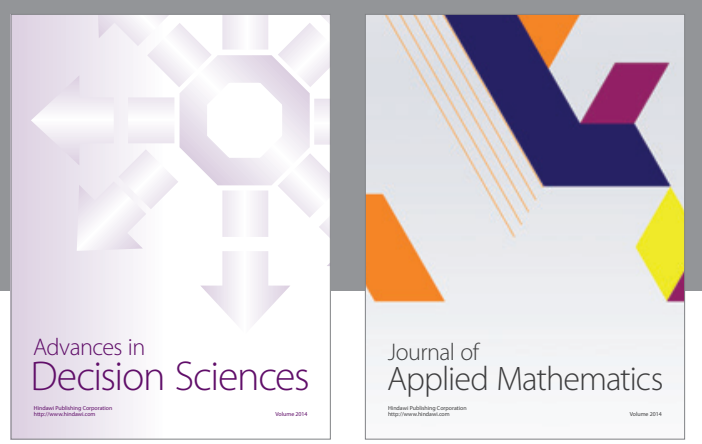

Algebra

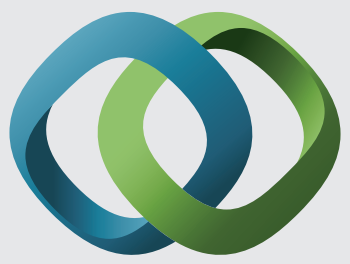

\section{Hindawi}

Submit your manuscripts at

http://www.hindawi.com
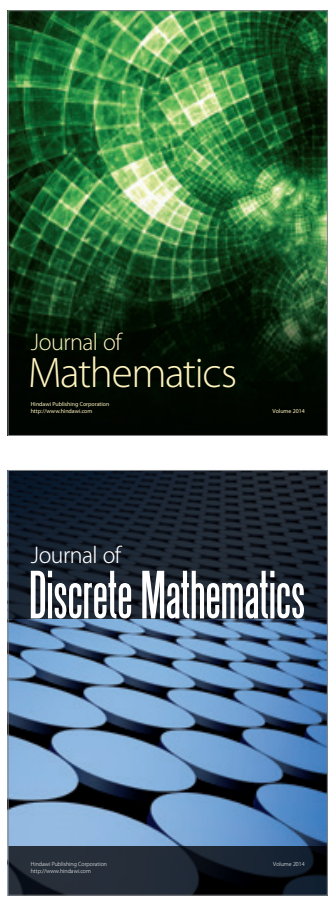

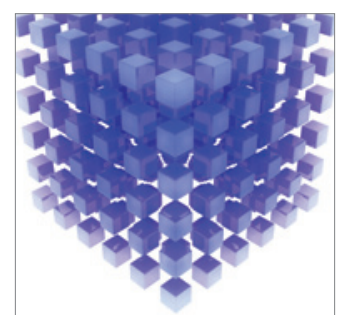

Mathematical Problems in Engineering
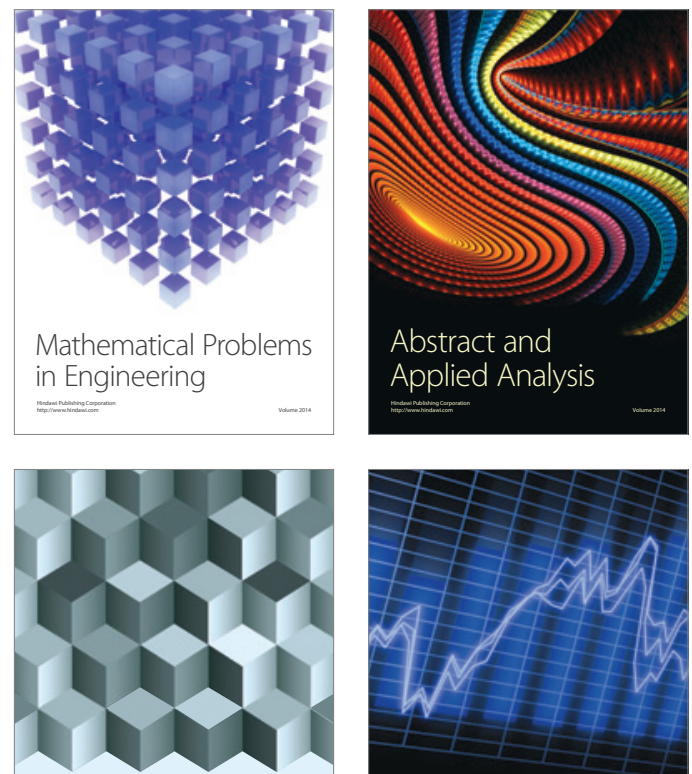

Journal of

Function Spaces

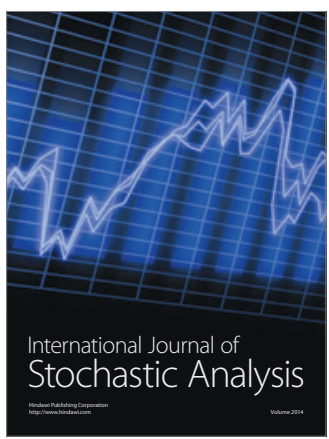

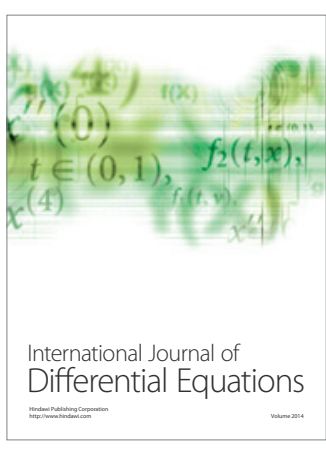
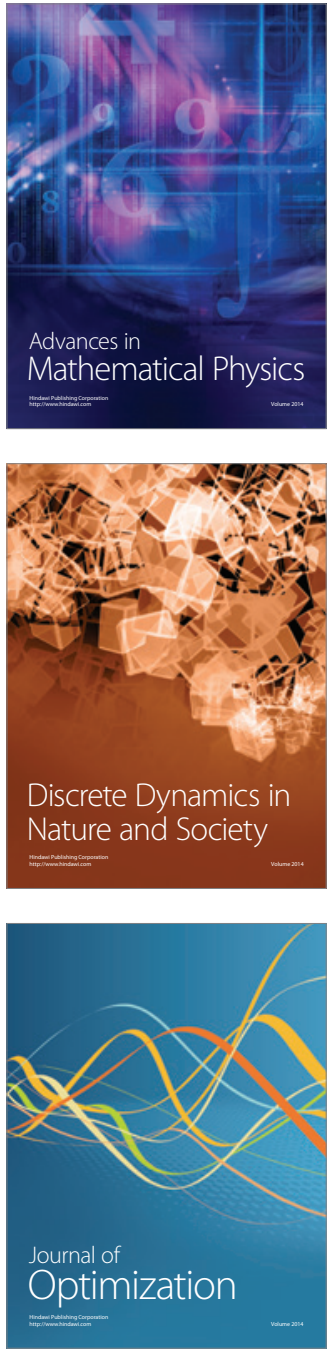